\title{
TRAJECTORIAL MARKET MODELS: ARBITRAGE AND PRICING INTERVALS
}

\author{
SEBASTIÁN E. FERRANDO, ALFREDO L. GONZÁLEZ, IVÁN L. DEGANO, \\ AND MASSOOMEH RAHSEPAR
}

\begin{abstract}
The paper develops general, non-probabilistic market models based on trajectory sets and minmax price bounds leading to price intervals for European options. The approach provides the trajectory based analogue of a martingale process as well as a generalization that allows a limited notion of arbitrage in the market while still providing coherent option prices. An illustrative example is described in detail. Several properties of the price bounds are obtained, in particular a connection with risk neutral pricing is established for trajectory markets associated to a continuous-time martingale model.
\end{abstract}

\section{INTRODUCTION}

The present paper provides a self contained framework for financial markets centered on a trajectory set modeling a risky asset. No probability measures, filtrations, or topological assumptions are required on this space. The approach singles out local trajectory properties that can be used to consistently build an associated option price theory. Such properties have already made their appearance in the stochastic literature ([11, 4, 15]).

By way of motivation, we mention that a recent trend in the mathematical finance literature develops results that weaken, or eliminate entirely, stochastic assumptions. For example, there are robust versions of the Fundamental Theorem of Asset Pricing (FTAP) in [20, 6, 7, 8, and [9]. To clarify the framework and provide perspective for the results in our paper, we describe natural connections with the new robust literature as well as with stochastic concepts. Our approach formalizes and generalizes the original model introduced by Britten-Jones and Neuberger $(\mathrm{BJ} \& \mathrm{~N})$ in [5, along the way we uncover some new phenomena not noticed there.

For simplicity, we model a one dimensional risky asset and rely on the availability of a risk-free bank account (both frictionless). The case of an arbitrary number of risky assets is developed in 12 and will be published elsewhere. The set of trajectories $\mathcal{S}$ is given by sequences of real numbers $S=\left\{S_{i}\right\}$, with $S_{0}=s_{0}$ for all

2010 Mathematics Subject Classification. 91G10; 91G20; 49J35; 49L20; 60G44.

Key words and phrases. Trajectory based market models; Arbitrage; Minmax; Martingales.

The research of S. E. Ferrando is supported in part by an NSERC grant. 
$S \in \mathcal{S} . \mathcal{H}$ is a family of investment strategies, $H=\left\{H_{i}\right\} \in \mathcal{H}$ is a sequence of functions acting on $\mathcal{S}$, representing the portfolio holdings $H(S)=\left\{H_{i}(S)\right\}$ along $S$; we assume $H_{i}(S)=H_{i}\left(S_{0}, \ldots, S_{i}\right)$. The set of trajectories $\mathcal{S}$ plays a central role in our work; trajectories, as opposed to probabilities, are a basic observable phenomena, therefore, it is relevant to pursue developments based only on such characteristics of the market. The models are discrete in the sense that we index potential portfolio rebalances, $H_{i}(S) \rightarrow H_{i+1}(S)$, by integer numbers. The total number of portfolio updates along a trajectory $S$ can be unbounded as a function of $S$. Otherwise, stock charts and investment amounts can take values in general subsets of the real numbers, data could flow in a time continuous manner and portfolio rebalances could be triggered by arbitrary events without the need to be associated to a time variable.

For a given European option $Z$, we introduce sub and super replication bounds for its price through a min-max operator. We study conditions on the market $\mathcal{M}=\mathcal{S} \times \mathcal{H}$ under which there exists a pricing interval $[\underline{V}(Z), \bar{V}(Z)]$. A general condition implying such result is called 0-neutral (Definition 8); it indicates that the price of the null function $Z=0$ is zero. General and natural local conditions on the trajectory space are required that ensure the existence of a price interval.

0 -neutrality is a weakening of no-arbitrage (Corollary 3 ) that allows for rational prices without any logical contradictions and with a basic financial supporting argument (the basic arguments are presented in Section 3). The co-existence of arbitrage and the price interval follows as a consequence of a worst case point of view and reflects a basic financial situation. Market players involved in the option's transaction may need/prefer the option's contract sure benefits against the potential arbitrage rewards.

Much of the recent literature on robust modeling has concentrated on dual representations of the nonlinear minmax operator $\bar{V}(\cdot)$ (given in Definition 5 (e.g. [7, 8, 9, 19, 20]). By way of contrast, our work emphasizes in Section 4 how characteristics of $\bar{V}(\cdot)$ and $\underline{V}(\cdot)$ depend on natural properties of the trajectory set.

Stochastic versions of the FTAP as well as robust versions have to be placed in the right perspective to appreciate our contributions. Reference [11] proves, in an arbitrary probability space with finite/discrete time, the equivalence of the three statements: a) No arbitrage; b) a local geometrical condition on the path of the process; c) existence of an equivalent martingale measure. The alluded condition b) can be described by:

$$
\text { if } Q\left(\Delta_{t} X>0 \mid \mathcal{F}_{t}\right)>0 \text { then } Q\left(\Delta_{t} X<0 \mid \mathcal{F}_{t}\right)>0 \text { as well, }
$$

or

$$
\text { if } Q\left(\Delta_{t} X>0 \mid \mathcal{F}_{t}\right)=0 \text { then } Q\left(\Delta_{t} X<0 \mid \mathcal{F}_{t}\right)=0 \text { as well, }
$$

where $X_{t}$ is an adapted process with respect to filtration $\mathcal{F}_{t}$. Our Definition 10 is a trajectorial version of (1.1). The latter condition is not emphasized on most versions of stochastic FTAP; this state of affairs is understandable as c) already characterizes the stochastic models of interest. Robust versions of FTAP emphasize analogous connections, namely dual representations which, assuming no arbitrage, connect super and under replication with martingale measures ([7, 9, 20, ). On the 
other hand, there is also the basic task of characterizing trajectory sets with simple and general assumptions that guarantee no arbitrage in a trajectory based market. That is, it is of interest to establish the relationships between a) and b) above in a non probabilistic setting, this is achieved, among other results, in the present paper.

A trajectory set is implicit in a stochastic process model; making trajectory sets a central object of interest is of relevance, in particular, when there is insufficient information to assign a probability distribution with confidence. An example is given by the modelling of crashes in [14] where, the number, timing and size of a downwards stock change (a crash) is treated without probabilistic assumptions. More importantly, giving trajectory sets a primary role changes the usual paradigm to model financial situations. Stochastic modeling relies on stochastic processes and the main input for their construction is a probability distribution; by contrast, the properties of their paths result as a by-product. References [2] and [3] present continuous-time examples of trajectory sets which do not correspond to semimartingales. In the present paper we describe a general discrete example that naturally incorporates trajectory dependent volatility. A computational and more detailed analysis of related examples is developed in [13. The latter reference also provides a detailed justification of an algorithm that evaluates the price bounds introduced in the present paper. Section 6]also introduces trajectory sets associated to continuous-time martingale processes.

A summary of the paper contents is provided next. Section 2 introduces the basic definitions. Section 3 proves existence of a price interval under general 0neutrality conditions; that section also compares the price interval with Merton's bounds and explains the meaning of the pricing interval when the market allows for arbitrage. Section 4 shows how local trajectory hypotheses lead to 0-neutral and no arbitrage markets as well as to existence of a price interval under specific assumptions. Section 5 deals with attainable functions, a generalization of this notion and some implications. Some analogues of martingale-like results are proven: in a 0-neutral market, today's stock price is the minmax price of future stock prices. Section 6 studies a general trajectory based market associated to a continuous-time martingale market model and draws connections between the introduced bounds and risk neutral pricing. Section 7 concludes. Appendix A describes an example illustrating the framework. Other appendices collect further results, connections to the robust literature, proofs, as well as some technical results needed in the main body of the paper.

\section{General, trajectory based models}

The paper concentrates on market models centered in properties of a given trajectory set. An example is given in Apendix A illustrating a general approach to constructing trajectory sets without using a priori probabilistic assumptions. 


\subsection{General setting.}

Definition 1 (Trajectory Set). Given a real number $s_{0}$, a set of trajectories $\mathcal{S}=$ $\mathcal{S}\left(s_{0}\right)$ is a subset of the set

$$
\mathcal{S}_{\infty}=\mathcal{S}_{\infty}\left(s_{0}\right)=\left\{S=\left\{S_{i}\right\}_{i \geq 0}: S_{i} \in \mathbb{R}, S_{0}=s_{0}\right\} .
$$

Definition 2 (Portfolio Set). A portfolio $H$ is a sequence of (pairs of) functions $H=\left\{\Phi_{i}=\left(B_{i}, H_{i}\right)\right\}_{i \geq 0}$ with $B_{i}, H_{i}: \mathcal{S} \rightarrow \mathbb{R}$, where $\mathcal{S} \subseteq \mathcal{S}_{\infty}\left(s_{0}\right) . H$ is said to be self-financing at $S \in \mathcal{S}$ if for all $i \geq 0$

$$
H_{i}(S) S_{i+1}+B_{i}(S)=H_{i+1}(S) S_{i+1}+B_{i+1}(S) .
$$

A portfolio $H$ is called non-anticipative if, for all $S, S^{\prime} \in \mathcal{S}$ satisfying $S_{k}^{\prime}=S_{k}$ for all $0 \leq k \leq i$, the equality $\Phi_{i}(S)=\Phi_{i}\left(S^{\prime}\right)$ holds.

Definition 3 (Trajectory Based Market). For a given real number $s_{0}$, a set of trajectories $\mathcal{S} \subseteq \mathcal{S}_{\infty}\left(s_{0}\right)$ and a set of portfolios $\mathcal{H}$, a trajectory based discrete market $\mathcal{M}=\mathcal{S} \times \mathcal{H}$ satisfies the following properties:

(1) For each $(S, H) \in \mathcal{M}$ there exists an integer $N=N_{H}(S)$, such that $\left[H_{k}(S)=H_{N-1}(S), \forall k \geq N_{H}(S)\right]$ or $\left[H_{k}(S)=H_{N}(S)=0, \forall k \geq N_{H}(S)\right]$.

(2) For $(S, H) \in \mathcal{M}, H$ is non-anticipative and self-financing at $S$.

Let $H=0=\left\{\left(0_{i}, 0_{i}\right)\right\}_{i \geq 0}$ (where $0_{i}$ is the function $0_{i}(S)=0$ ) denote the 0 portfolio. We will also assume $\{H=0\} \in \mathcal{H}$, with $N_{0} \equiv 0$; also, if $H \neq 0$ then $N_{H} \geq 1$. Any such $\mathcal{M}$ will be referred to as a market in the rest of the paper,

Our results are also valid if one restricts $S_{i}$ to belong to a given apriori set of values and so, reflecting the actual discrete nature of stock chart values (for example, values of $S_{i}$ could be represented by a finite number of decimal digits). Similarly, the values $H_{i}(S)$ can belong to an arbitrary fixed subset of $\mathbb{R}$, for example, integer multiples of a given real number.

$H_{k}(S)=H_{N-1}(S)$ for all $k \geq N_{H}(S)$ means rebalancing stops at, or prior to, $N_{H}(S)-1$. The condition $H_{k}(S)=H_{N}(S)=0$ for all $k \geq N_{H}(S)$ means definite liquidation has taken place at, or prior to, $N_{H}(S)$; such portfolio will be referred to as liquidated. In the case of pricing a European option maturing at time $T, S_{N_{H}(S)}$ is interpreted as $x(T)$ (i.e., as a possible stock chart value at time $T$ ).

Section 2.2 extends the above setting to account for other sources of uncertainty, accommodating this extension is mostly a matter of notation and, hence, most of the paper will only employ the above introduced notation.

For simplicity, we denote $\Delta_{i} S \equiv S_{i+1}-S_{i}$. Given $(S, H) \in \mathcal{M}$, the self-financing property 2.1 implies that the portfolio value, defined by $V_{H}(i, S)=B_{i}(S)+$ $H_{i}(S) S_{i}$, where $B_{i}(S)$ represent the holdings in a bank account, equals:

$$
V_{H}(i, S)=V_{H}\left(0, S_{0}\right)+\sum_{k=0}^{i-1} H_{k}(S) \Delta_{k} S,
$$

during the period $[i, i+1)$ for $i=0, \ldots, N_{H}(S)-1$ and valid over $\left[N_{H}(S), \infty\right)$ for the case $i \geq N_{H}(S)$. Of course, $V_{H}\left(0, S_{0}\right) \equiv V_{H}(0, S)=B_{0}(S)+H_{0}(S) S_{0}$.

Observe that we assume zero interest rates of the bank account and that there are no transaction costs. 
Remark 1. As defined above, a portfolio $H$ is given by specifying the pairs of functions $\left\{\left(B_{i}, H_{i}\right)\right\}$ so that 2.1 holds. In the remaining of the paper, we will define $H$ more conveniently by specifying the non-anticipative functions $H_{i}$ and an initial portfolio value $V_{0}=V_{H}\left(0, S_{0}\right)$; this will provide $B_{0}$, and the remaining functions $B_{i}, i \geq 1$, are then obtained by solving equations (2.1).

The introduced framework is quite general; notice that nothing requires $H_{i}(S) \neq$ $H_{i+1}(S) . S_{i+1}$ is the stock value at which investors $H \in \mathcal{H}$, that have invested so far $H_{k}(S), 0 \leq k \leq i$, may rebalance their holdings to $H_{i+1}(S)$.

The following conditional spaces will play a key role. Given $\mathcal{M}, S \in \mathcal{S}$ and $k \geq 0$ fixed, set

$$
\mathcal{S}_{(S, k)} \equiv\left\{\tilde{S} \in \mathcal{S}: \tilde{S}_{i}=S_{i}, 0 \leq i \leq k\right\} .
$$

Unless specified otherwise, $Z$ denotes a general function $Z: \mathcal{S} \rightarrow \mathbb{R}$; pairs $(S, k)$ or triples $(S, k, H), S \in \mathcal{S}, k \geq 0, H \in \mathcal{H}$, will be referred to as nodes.

Some results require that the functions $N_{H}: \mathcal{S} \rightarrow \mathbb{N}$, introduced in Definition 3 are stopping times, according to the following definition.

Definition 4 (Trajectory Based Stopping Times). Given a trajectory space $\mathcal{S}$, a trajectory based stopping time (or stopping time for short) is a function $\nu: \mathcal{S} \rightarrow \mathbb{N}$ such that if $S, S^{\prime} \in \mathcal{S}$ and $S_{k}=S_{k}^{\prime}$, for $0 \leq k \leq \nu(S)$, then $\nu\left(S^{\prime}\right)=\nu(S)$.

We refer to [21] (see also [3]) for an account of the relationship between the above notion of trajectory based stopping time and filtration based stopping times. Such $\nu$ will be simply referred to as a stopping time in the remaining of the paper.

2.2. Other sources of uncertainty. All results and definitions in the paper involving markets $\mathcal{M}$ and trajectory sets $\mathcal{S}$ can be generalized by incorporating another source of uncertainty besides the stock. This extra source of uncertainty will be denoted by $W=\left\{W_{i}\right\}$ which, in financial terms, will be considered to be an observable quantity. This is analogous to moving from the natural filtration to an augmented filtration in the stochastic setting.

The sequence elements $W_{i}$ are assumed to belong to abstract sets $\Omega_{i}$ from which we only require to have defined an equality relationship. We provide next the simple changes to the previous definitions to accommodate for the new source of uncertainty. The arrow notation $\rightarrow$ indicates how the objects change $\left(\left(s_{0}, w_{0}\right)\right.$ is fixed).

$$
\begin{aligned}
& \mathcal{S}_{\infty}\left(s_{0}\right) \rightarrow \mathcal{S}_{\infty}^{\mathcal{W}}\left(s_{0}, w_{0}\right) \equiv\left\{\mathbf{S}=\left\{\mathbf{S}_{i} \equiv\left(S_{i}, W_{i}\right)\right\}_{i \geq 0}: W_{i} \in \Omega_{i},\left(S_{0}, W_{0}\right)=\left(s_{0}, w_{0}\right)\right\} \\
& \mathcal{S}=\mathcal{S}\left(s_{0}\right) \rightarrow \mathcal{S}^{\mathcal{W}} \equiv \mathcal{S}^{\mathcal{W}}\left(s_{0}, w_{0}\right) \subseteq \mathcal{S}_{\infty}^{\mathcal{W}}\left(s_{0}, w_{0}\right) . \\
& H_{i}(S) \rightarrow H_{i}(\mathbf{S}) \\
& \mathcal{S}_{(S, k)} \rightarrow \mathcal{S}_{(\mathbf{S}, k)}^{\mathcal{W}}\left(s_{0}, w_{0}\right) \equiv\left\{\tilde{\mathbf{S}} \in \mathcal{S}^{\mathcal{W}}\left(s_{0}, w_{0}\right), \tilde{\mathbf{S}}_{i}=\mathbf{S}_{i}, 0 \leq i \leq k\right\} \\
& V_{H}(k, S) \rightarrow V_{H}(k, \mathbf{S})=V_{H}\left(0,\left(S_{0}, w_{0}\right)\right)+\sum_{i=0}^{k-1} H_{i}(\mathbf{S}) \Delta_{i} S .
\end{aligned}
$$


Besides the above changes, that concern mostly trajectory sets and the functional dependency $H_{i}\left(\right.$ ) in terms of both variables $S_{k}, W_{k}$ (and some minor notational changes), all statements and properties appearing in the paper, only involve the first coordinate $S_{i}$ (in the tuples $\left(S_{i}, W_{i}\right)$ ) in all algebraic manipulations. Clearly, $H_{i}$ is required to be non-anticipative with respect two both variables $S_{k}$ and $W_{k}$ and the notion of trajectory based stopping time applies now to trajectories of the form $\mathbf{S}=\left\{\left(S_{i}, W_{i}\right)\right\}$. These remarks can be used to show that all the results in the paper stay true in the extended/augmented formalism. We explicitly use the extended formalism in Subsection A.1 and Section 6.

\section{Pricing With arbitrage in 0-Neutral markets}

The quantities $\bar{V}\left(S_{0}, Z, \mathcal{M}\right)$ and $\underline{V}\left(S_{0}, Z, \mathcal{M}\right)$ introduced below for our setting, correspond to the usual super and sub hedging prices, respectively, defined in robust frameworks (e.g. [6, 9, 20]) for a contingent claim (function) $Z: \mathcal{S} \rightarrow \mathbb{R}$.

We provide general conditions resulting in a worst case price interval for the possible prices for a European option. The notion of conditionally 0-neutral market (given in Definition 8) is the essential ingredient for the result to hold. We compare the minimax bounds with Merton's bounds, and give a detailed justification for the quantities introduced to be actual market prices. As already indicated, 0-neutrality is a weakening of the no arbitrage condition which allows a price interval to exists even when there is a certain kind of arbitrage opportunity in the market. See discussion after Corollary 3 in Section 4.

Definition 5 (Conditional Minmax Bounds). Given a market $\mathcal{M}=\mathcal{S} \times \mathcal{H}$ and a node $(S, k)$, define

$$
\bar{V}_{k}(S, Z, \mathcal{M}) \equiv \inf _{H \in \mathcal{H}} \sup _{\tilde{S} \in \mathcal{S}_{(S, k)}}\left[Z(\tilde{S})-\sum_{i=k}^{N_{H}(S)-1} H_{i}(\tilde{S})\left(\tilde{S}_{i+1}-\tilde{S}_{i}\right)\right] .
$$

Also $\underline{V}_{k}(S, Z, \mathcal{M}) \equiv-\bar{V}_{k}(S,-Z, \mathcal{M})$. Set $\bar{V}\left(S_{0}, Z, \mathcal{M}\right) \equiv \bar{V}_{0}(S, Z, \mathcal{M})$ and $\underline{V}\left(S_{0}, Z, \mathcal{M}\right) \equiv \underline{V}_{0}(S, Z, \mathcal{M})$ as well.

The quantity $\bar{V}_{k}(S, Z, \mathcal{M})$ is the minimum capital needed, conditional on a node $(S, k)$, to super-hedge $Z$. An analogous interpretation applies to $\underline{V}_{k}(S, Z, \mathcal{M})$. Sufficient conditions on $Z$ to guarantee finiteness of the minmax bounds are given in Subsection D.1

The notion of extended arbitrage (see [10, Def. 2.2] and [20, Def. 3.1]) introduced below is useful in order to partially justify the above minmax definitions as price bounds.

Definition 6 (Extended Arbitrage). Let $Z: \mathcal{S} \rightarrow \mathbb{R}$ be a function defined on $\mathcal{S}$ and $\mathcal{M}=\mathcal{S} \times \mathcal{H}$ a discrete market. $H \in \mathcal{H}$, with initial value $V_{0}$, is an extended arbitrage with respect to $Z$ if

$V_{0}+\sum_{i=0}^{N_{H}(S)-1} H_{i}(S) \Delta_{i} S-Z(S) \geq 0 \forall S \in \mathcal{S}$, and strictly positive for some $S^{*} \in \mathcal{S}$, 
or $Z(S)-V_{0}+\sum_{i=0}^{N_{H}(S)-1} H_{i}(S) \Delta_{i} S \geq 0 \forall S \in \mathcal{S}$, and strictly positive for some $S^{*} \in \mathcal{S}$.

For a comparison with the definition below, see [10].

Definition 7 (Fair Price). We say that $\pi$ is a fair price for a function $Z$ in a discrete market $\mathcal{M}=\mathcal{S} \times \mathcal{H}$ if there is no $H \in \mathcal{H}$, with initial value $V_{H}\left(0, S_{0}\right)=\pi$, which is an extended arbitrage with respect to $Z$.

Observe that neither $\pi>\bar{V}\left(S_{0}, Z, \mathcal{M}\right)$ nor $\pi<\underline{V}\left(S_{0}, Z, \mathcal{M}\right)$ are fair prices for $Z$. Theorem 1 below provides conditions under which the fair prices are confined to an interval (see Remark 2 in Section 3.1), as it is known for stochastic models which are arbitrage free.

The following proposition shows that in a general discrete market the quantities $\bar{V}\left(S_{0}, Z, \mathcal{M}\right), \underline{V}\left(S_{0}, Z, \mathcal{M}\right)$ may behave in an unexpected way, but not so in a market which satisfies the 0-neutral property (introduced below).

Definition 8 (Conditionally 0 -Neutral). We say that a discrete market $\mathcal{M}$ is conditionally 0 -neutral at node $(S, k)$ if

$$
\bar{V}_{k}(S, Z=0, \mathcal{M})=0 .
$$

For $k=0$, the conditional 0-neutral property, which depends on $S$ only through $S_{0}$, will be referred to as 0 -neutral. That notion has not taken central stage in the literature; we borrowed it from [5], where it was considered equivalent to noarbitrage, while in Section 4.2 we make the point that arbitrage opportunities are allowed under such assumption.

Proposition 1. Given a discrete market model $\mathcal{M}=\mathcal{S} \times \mathcal{H}$ and $c$ an arbitrary constant, it follows that

$$
\text { if } Z(S)=c \text { for all } S \in \mathcal{S} \text {, then } \bar{V}\left(S_{0}, Z, \mathcal{M}\right) \leq c \leq \underline{V}\left(S_{0}, Z, \mathcal{M}\right) \text {. }
$$

In contrast, notice that if $\mathcal{M}$ is 0 -neutral then $\bar{V}\left(S_{0}, Z, \mathcal{M}\right)=c=\underline{V}\left(S_{0}, Z, \mathcal{M}\right)$.

Proof. Consider first the case that $\mathcal{M}$ is not 0-neutral; so $\bar{V}\left(S_{0}, \mathbf{0}, \mathcal{M}\right)<0$ in that case, this implies that there exists $H \in \mathcal{H}$ such that

$$
-\sum_{i=0}^{N_{H}(S)-1} H_{i}(S) \Delta_{i} S<0, \quad \text { for all } S \in \mathcal{S},
$$

so

$$
\sup _{S \in \mathcal{S}}\left[c-\sum_{i=0}^{N_{H}(S)-1} H_{i}(S) \Delta_{i} S\right] \leq c,
$$

which leads to $\bar{V}\left(S_{0}, \mathbf{c}, \mathcal{M}\right) \leq c$.

Consider now that $\mathcal{M}$ is 0 -neutral; it is then clear that $\bar{V}\left(S_{0}, \mathbf{c}, \mathcal{M}\right)=c=$ $\underline{V}\left(S_{0}, \mathbf{c}, \mathcal{M}\right)$. Hence $\bar{V}\left(S_{0}, \mathbf{c}, \mathcal{M}\right) \leq c$ in all cases, then $\underline{V}\left(S_{0}, \mathbf{c}, \mathcal{M}\right)=$ $-\bar{V}\left(S_{0},-\mathbf{c}, \mathcal{M}\right) \geq c$. 
The next theorem provides conditions under which there exists a price interval. The definition of addition of two portfolios, implicitly required in it, is introduced just before the statement of Lemma 2 in Appendix D

Theorem 1. Consider a discrete market $\mathcal{M}=\mathcal{S} \times \mathcal{H}$ and a function $Z$ defined on $\mathcal{S}$. Assume $\mathcal{M}$ is conditionally 0 -neutral at node $(S, k)$ and $\mathcal{H}$ is closed under addition, then

$$
\underline{V}_{k}(S, Z, \mathcal{M}) \leq \bar{V}_{k}(S, Z, \mathcal{M})
$$

Alternatively, $(3.2$ also holds if $\mathcal{S} \times(\mathcal{H}+\mathcal{H})$ is conditionally 0 -neutral at node $(S, k)$ and, furthermore, either $N_{H}$ is a stopping time for all $H \in \mathcal{H}$ or all $H \in \mathcal{H}$ are liquidated.

Proof. Taking $\mathcal{H}^{1}=\mathcal{H}^{2}=\mathcal{H}$, and having in mind that the sum of portfolios is guaranteed by either hypothesis, the result follows directly from the conclusion (D.3) of Lemma 2 in Appendix D

Section 4 gives general conditions guaranteeing the key condition of 0-neutrality needed in Theorem 1. Proposition 5 in that section, shows that 0-neutrality is a weakening of no-arbitrage. Notice that assuming $\mathcal{S} \times(\mathcal{H}+\mathcal{H})$ to be conditionally 0 -neutral at node $(S, k)$ implies $\mathcal{M}$ to be conditionally 0 -neutral at that node as well. Assuming the stronger hypothesis $\mathcal{H}+\mathcal{H}=\mathcal{H}$ is not necessary, as it is clearly shown by Corollary 2 in Section 4 , which provides assumptions implying the conditional 0 -neutral property as well as 3.2 without requiring $\mathcal{H}$ to be closed under addition.

The following is another condition on $\mathcal{S}$ that also ensures 3.2 ; the proof is immediate and so omitted.

Proposition 2. Consider a market $\mathcal{M}=\mathcal{S} \times \mathcal{H}$, a function $Z$ defined on $\mathcal{S}$, a fixed $S \in \mathcal{S}$ and $k \geq 0$. If there exists a sequence $S^{0} \in \mathcal{S}_{(S, k)}$ such that $S_{i}^{0}=S_{k}$ for all $i \geq k$, then $\mathcal{M}$ is conditionally 0 -neutral at $(S, k)$, and

$$
\underline{V}_{k}(S, Z, \mathcal{M}) \leq Z\left(S^{0}\right) \leq \bar{V}_{k}(S, Z, \mathcal{M}) .
$$

We provide next the simple connection between the minmax bounds and Merton's bounds [18. For a call option $C_{K}(x)=(x-K)_{+}$, with $K>0$, Merton's bounds are $C_{K}\left(S_{0}\right)$ and $S_{0}$.

Proposition 3 (Merton's Bounds Comparison). Consider a market $\mathcal{M}$, an integer valued function $N=N(S), S \in \mathcal{S}$, and a function $Z$ defined on $\mathcal{S}$. Assume there exists $H^{ \pm} \in \mathcal{H}$ such that $H_{i}^{ \pm}(S)= \pm 1$ and for any $S \in \mathcal{S}$ and $0 \leq i \leq N_{H}(S) \equiv$ $N(S)$. We obtain:

(a) If $Z(S)=C_{K}\left(S_{N}\right)$ and $\mathcal{M}$ is 0 -neutral, then $C_{K}\left(S_{0}\right) \leq \underline{V}\left(S_{0}, Z, \mathcal{M}\right)$.

(b) If $Z(S) \leq S_{N}$ for all $S \in \mathcal{S}, \bar{V}\left(S_{0}, Z, \mathcal{M}\right) \leq S_{0}$.

Proof. Fix $S \in \mathcal{S}, 0$-neutrality implies $\underline{V}\left(S_{0}, Z, \mathcal{M}\right) \geq 0$, so (a) is clearly valid if $S_{0} \leq K$. If $S_{0}>K$

$$
C_{K}\left(S_{0}\right)=S_{0}-K \leq\left(S_{N}-K\right)_{+}-\left(S_{N}-S_{0}\right)=Z(S)+\sum_{i=0}^{N-1}-\Delta_{i} S .
$$


Thus, $C_{K}\left(S_{0}\right) \leq \inf _{S \in \mathcal{S}}\left[Z(S)-\sum_{i=0}^{N-1} \Delta_{i} S\right] \leq \underline{V}\left(S_{0}, Z, \mathcal{M}\right)$.

(b) $S_{N}-S_{0}=\sum_{i=0}^{N-1} \Delta_{i} S$, then $S_{0}=S_{N}-\sum_{i=0}^{N-1} \Delta_{i} S \geq Z(S)-\sum_{i=0}^{N-1} \Delta_{i} S$. Consequently $S_{0} \geq \sup _{S \in \mathcal{S}}\left[Z(S)-\sum_{i=0}^{N-1} \Delta_{i} S\right] \geq \bar{V}\left(S_{0}, Z, \mathcal{M}\right)$.

In a situation where Proposition 2 and Proposition 3 , item a), are both applicable, we obtain the interesting result $C_{K}\left(S_{0}\right)=\underline{V}\left(S_{0}, Z, \mathcal{M}\right)$. This shows that a characteristic of the trajectory space, namely the presence of a globally constant trajectory, implies that the lower Merton bound is attained.

We refer to Appendix $\mathrm{D}$ for complementary material.

3.1. Meaning of option prices in 0-neutral markets. Having in mind the assumptions leading to the conclusion $\underline{V}(S, Z, \mathcal{M}) \leq \bar{V}(S, Z, \mathcal{M})$ (as in Theorem 1 . Proposition 2 , and Corollary 2, we introduce the following definition of price interval.

Definition 9. Consider a market $\mathcal{M}=\mathcal{S} \times \mathcal{H}$ and a function $Z$ on $\mathcal{S}$. Under the assumption that $\underline{V}\left(S_{0}, Z, \mathcal{M}\right) \leq \bar{V}\left(S_{0}, Z, \mathcal{M}\right)$, we will call $\left[\underline{V}\left(S_{0}, Z, \mathcal{M}\right), \bar{V}\left(S_{0}, Z, \mathcal{M}\right)\right]$ the price interval of $Z$ relative to $\mathcal{M}$.

Remark 2. Observe that under the referred assumptions

$$
\pi \in\left(\underline{V}\left(S_{0}, Z, \mathcal{M}\right), \bar{V}\left(S_{0}, Z, \mathcal{M}\right)\right)
$$

is a fair price for $Z$.

The assumptions in Theorem 1 guarantee a pricing interval and at the same time allow for arbitrage in the market. See Section 4.2, Corollary 3 and discussion afterwards. It should be noted that the presence of arbitrage nodes (see Section 4 ) will impact the actual value of the option bounds. Examples for the extent to which this could happen are documented in [13].

Under the assumption that an option contract has been traded, the existence of the minmax price interval, independently of the presence of an arbitrage strategy, is substantiated on the need to have enough funds to match the certainty of future financial obligations. This is in contrast to an investment in an arbitrage opportunity (see Definition 12 which profits are uncertain as they may not materialize in a 0-neutral market. In a general 0-neutral market, an investment following an arbitrage portfolio will not guarantee enough returns under all scenarios in order to cover the obligations required by $Z$.

The simplest mathematical example illustrating such a financial situation is given by a one-step market $\mathcal{M}$ where $N_{H}(S)=1$ for all $(S, H), \sup _{\tilde{S} \in \mathcal{S}}\left(\tilde{S}_{1}-s_{0}\right)>0$ and $\inf _{\tilde{S} \in \mathcal{S}}\left(\tilde{S}_{1}-s_{0}\right)=0$ is realized at a unique $\hat{S} \in \mathcal{S}$. So, the market is 0-neutral. Furthermore, if $\left\{H_{0}(S): S \in \mathcal{S}, H \in \mathcal{H}\right\}=\mathbb{R}$ one can also see that $\bar{V}\left(s_{0}, Z, \mathcal{M}\right)=$ $Z\left(\hat{S}_{1}\right)$ where $Z$ is a European call option. The minmax price provides a solution reflecting the needs of investors dealing with the option. Namely, if the option selling price is smaller than $\bar{V}\left(s_{0}, Z, \mathcal{M}\right)$ the potential obligation $Z\left(S_{1}\right)$ could not be matched under all scenarios through investing on the arbitrage (as actual profits 
may not materialize) resulting in a shortage of funds, under some scenarios, for the seller of the option. So, it is the worst case approach, requiring coverage under all scenarios, that allows for the co-existence of arbitrage and a price interval in a 0 -neutral market. If 0 -neutrality does not hold, it is easy to see that the minmax optimization falls back into the arbitrage opportunity by giving $\bar{V}\left(s_{0}, Z, \mathcal{M}\right)=-\infty$ and the optimal investment $h$ given by $h^{*}=\infty$ in the above example.

See also related arguments in [16] where, in a context of portfolio selection, a numeraire portfolio is shown to exist under conditions that allow for arbitrage opportunities.

\section{TRAJECTORY BASED CONDITIONS FOR 0-NEUTRAL MARKETS}

Theorem 1 in Section 3 shows the key role of conditional 0-neutrality in order to obtain a worst case price interval. The present section provides natural and general sufficient conditions that imply a market $\mathcal{M}$ to be conditionally 0-neutral. The key ingredients are the local (i.e., relative to a given node) path conditions, introduced in Definition 10, that allow trajectories to move in a contrarian way to an arbitrary investment. These are a trajectorial version of properties satisfied by a martingale process. There is also a need for global conditions related to how market participants may stop their portfolio rebalances. We provide a general financial setting leading to one such global condition, these assumptions also imply existence of a price interval.

Definition 10 (Local Conditions: 0-Neutral \& Arbitrage-Free Nodes). Given a trajectory space $\mathcal{S}$ and a node $(S, j)$ :

- $(S, j)$ is called a 0-neutral node if

$$
\sup _{\tilde{S} \in \mathcal{S}(S, j)}\left(\tilde{S}_{j+1}-S_{j}\right) \geq 0 \quad \text { and } \quad \inf _{\tilde{S} \in \mathcal{S}(S, j)}\left(\tilde{S}_{j+1}-S_{j}\right) \leq 0 .
$$

- $(S, j)$ is called an arbitrage-free node if

$$
\sup _{\tilde{S} \in \mathcal{S}(S, j)}\left(\tilde{S}_{j+1}-S_{j}\right)>0 \quad \text { and } \inf _{\tilde{S} \in \mathcal{S}(S, j)}\left(\tilde{S}_{j+1}-S_{j}\right)<0
$$

or

$$
\sup _{\tilde{S} \in \mathcal{S}(S, j)}\left(\tilde{S}_{j+1}-S_{j}\right)=\inf _{\tilde{S} \in \mathcal{S}(S, j)}=0=\left(\tilde{S}_{j+1}-S_{j}\right) .
$$

$\mathcal{S}$ is called locally 0 -neutral if every node is 0 -neutral. $\mathcal{S}$ is said to be locally arbitrage-free if every node is arbitrage free. A node that satisfies 4.2 will be called an up-down node, and a node satisfying 4.3 will be called a flat node. A node that is 0 -neutral but that is not an arbitrage-free node, will be called an arbitrage node.

An arbitrage-free node is clearly a 0 -neutral node as well. If all nodes $(\tilde{S}, k)$, $\tilde{S} \in \mathcal{S}_{(S, j)}$ and $k \geq j$, are 0 -neutral and $\mathcal{H}$ is a set of portfolios, it follows that $\inf _{H \in \mathcal{H}}\left\{\sup _{\tilde{S} \in \mathcal{S}_{(S, j)}}\left[-H_{j}(S) \Delta_{j} \tilde{S}\right]\right\}=0$.

The following definition contains a main tool: it introduces a trajectory that for a given investment provides arbitrarily small profits. 
Definition 11 ( $\epsilon$-contrarian). Given $H \in \mathcal{H}, S \in \mathcal{S}, \epsilon \geq 0$ and $n \geq 1$, if

$$
\exists S^{n, \epsilon} \in \mathcal{S}_{(S, n)} \quad \text { and } \quad \sum_{i=n}^{N_{H}\left(S^{n, \epsilon}\right)-1} H_{i}\left(S^{n, \epsilon}\right) \Delta_{i} S^{n, \epsilon}<\epsilon,
$$

we will say that $S^{n, \epsilon}$ is $\epsilon$-contrarian beyond $n$ w.r.t. $(S, H)$.

Notice that $S^{n, \epsilon}=S$ trivially satisfies the above definition for the case $n \geq$ $N_{H}(S)$ and $\epsilon>0$.

The following is a useful characterization of 0-neutrality of the market, which follows directly from the corresponding definitions, connecting it with local properties of the trajectories.

Proposition 4. $\mathcal{M}$ is conditionally 0 -neutral at node $(S, k)$ if and only if for each $H \in \mathcal{H}$ and $\epsilon>0$, there exists $S^{k, \epsilon}$ which is $\epsilon$-contrarian beyond $k$ w.r.t. $(S, H)$.

The local 0-neutral property of $\mathcal{S}$ makes it possible to obtain trajectories which are almost $\epsilon$-contrarian, this is shown in the next lemma.

Lemma 1. Given a market $\mathcal{M}=\mathcal{S} \times \mathcal{H}, k \geq 0, S^{k} \in \mathcal{S}$, and $H \in \mathcal{H}$. Assume each node $(S, j)$, with $S \in \mathcal{S}_{\left(S^{k}, k\right)}$ and $j \geq k$, is 0-neutral. Then, for any $\epsilon>0$, there exists a sequence of trajectories $\left(S^{m}\right)_{m \geq k}$ satisfying

$$
S^{m} \in \mathcal{S}_{\left(S^{m-1}, m-1\right)}, \quad \text { and } \quad \sum_{i=k}^{n-1} H_{i}\left(S^{m}\right) \Delta_{i} S^{m}<\sum_{i=k}^{n-1} \frac{\epsilon}{2^{i}} \leq \epsilon, \quad \text { for } m \geq n>k .
$$

Proof. Fix $\epsilon>0$. By the assumed 0-neutral property of the nodes, there exists $S^{k+1} \in \mathcal{S}_{\left(S^{k}, k\right)}$ such that

$$
H_{k}\left(S^{k+1}\right) \Delta_{k} S^{k+1}<\frac{\epsilon}{2^{k}} .
$$

Recursively, for $m \geq k+1$, once $S^{m} \in \mathcal{S}_{\left(S^{m-1}, m-1\right)}$ was chosen, satisfying 4.6, with $m-1$ taking the place of $k$, and then

$$
\sum_{i=k}^{m-1} H_{i}\left(S^{m}\right) \Delta_{i} S^{m}<\sum_{i=k}^{m-1} \frac{\epsilon}{2^{i}} \leq \epsilon
$$

there exists $S^{m+1} \in \mathcal{S}_{\left(S^{m}, m\right)}$ satisfying 4.6 with $k$ replaced by $m$, and 4.7) with $m$ replaced by $m+1$.

The local hypotheses, from Definition 10 are not sufficient to guarantee conditional (global) 0-neutrality of $\mathcal{M}$. The next corollary, requiring boundedness of $N_{H}$ for any $H \in \mathcal{H}$, is a hypothesis that, when added to the local 0-neutral property, guarantees that a market is 0-neutral. Boundedness of $N_{H}$ is a strong condition that is not necessary, this is made clear in Corollary 2 Other sufficient conditions for the existence of a price interval allowing $N_{H}$ to be unbounded are also possible; for reasons of space they are just sketched in Appendix C. 
Corollary 1. Given a market $\mathcal{M}=\mathcal{S} \times \mathcal{H}, k \geq 0, S^{k} \in \mathcal{S}$. Assume each node $(S, j)$, with $S \in \mathcal{S}_{\left(S^{k}, k\right)}$ and $j \geq k$, is 0 -neutral and $N_{H}$ is bounded for any $H \in \mathcal{H}$. Then $\mathcal{M}$ is conditionally 0 -neutral at node $(S, k)$.

Proof. Fix $H \in \mathcal{H}$ and $\epsilon>0$. By Lemma 1 there exist a sequence of trajectories $\left(S^{m}\right)_{m \geq k}$ for which 4.5 holds. Let $M$ a bound for $N_{H}$, then $S^{M}$ is $\epsilon$-contrarian beyond $k$ w.r.t. $\left(S^{k}, H\right)$. So, the conclusion is given by Proposition 4 .

4.1. Debt limited portfolios. Here we introduce a set of financially motivated hypotheses, of a general nature, that, when combined with the local 0-neutral (local arbitrage-free) assumption on $\mathcal{S}$, provide conditionally 0-neutral (arbitrage-free) markets $\mathcal{M}$. In fact, the following theorem shows that for all practical financial purposes, as long as the number of arbitrage and flat nodes are bounded along each trajectory, the assumption of existence of contrarian trajectories is always satisfied. The results rely on limiting the capital that a portfolio owner may be able to borrow; this condition is usually used to exclude arbitrage opportunities created by doubling strategies ([4). The setting allows for unbounded $N_{H}$.

The next theorem provides a natural and general setting, ensuring that a market is conditionally 0 -neutral.

Theorem 2. Given a market $\mathcal{M}=\mathcal{S} \times \mathcal{H}, S \in \mathcal{S}$, and $n \geq 0$. Assume each node $\left(S^{\prime}, j\right), S^{\prime} \in \mathcal{S}_{(S, n)}$ with $j \geq n$, is 0-neutral. We further assume:

(1) The number of arbitrage 0-neutral and flat nodes (as per Definition 10) allowed in each trajectory is bounded by an absolute constant $\hat{m}$. Also, for $H \in \mathcal{H}$ :

(2) There exists $A=A(H) \geq 0$, a constant independent of $S$ and $k$, satisfying:

$$
V_{0}+\sum_{i=0}^{k-1} H_{i}\left(S^{\prime}\right) \Delta_{i} S^{\prime} \geq-A, \quad 0 \leq k \leq N_{H}\left(S^{\prime}\right), \quad \forall S^{\prime} \in \mathcal{S}_{(S, n)} .
$$

(3) There exists $\delta$, a constant that may depend on $H$, satisfying:

$$
\forall S^{\prime} \in \mathcal{S}_{(S, n)} \text { and } i \geq n \text { such that }\left(S^{\prime}, i\right) \text { is an up-down node: }
$$

$$
\text { if } H_{i}\left(S^{\prime}\right) \neq 0 \text { then } \exists \hat{S} \in \mathcal{S}_{\left(S^{\prime}, i\right)} \text { such that }-H_{i}(\hat{S}) \Delta_{i} \hat{S} \geq \delta>0 \text {. }
$$

Then, for any $\epsilon>0$, there exists $S^{n, \epsilon} \in \mathcal{S}_{(S, n)}$ so that $H$ and $S^{n, \epsilon}$ are $\epsilon$-contrarian beyond $n$. In particular, if hypotheses (2) and (3) above are satisfied for all $H \in \mathcal{H}$, then $\mathcal{M}$ is conditionally 0 -neutral at $(S, n)$.

Item 1 above only allows a constant maximum $\hat{m}$ of arbitrage 0-neutral and flat nodes along each trajectory but, those nodes, are allowed to be arbitrarily distributed along such trajectory.

Proof. It is enough to consider the case $n<N_{H}\left(S^{\prime}\right)$ for any $S^{\prime} \in \mathcal{S}_{(S, n)}$. We will establish the existence of $S^{*} \in \mathcal{S}_{(S, n)}$ such that $-\sum_{i=n}^{N_{H}\left(S^{*}\right)-1} H_{i}\left(S^{*}\right) \Delta_{i} S^{*} \geq 0$, and this will conclude the proof.

Let $n_{0}$ be the smallest integer satisfying $n \leq n_{0}<N_{H}(S)$ and $H_{n_{0}}(S) \neq 0$. If such $n_{0}$ does not exist we take $S^{*} \equiv S$. There are two possibilities: a) $\left(S, n_{0}\right)$ is an 
arbitrage 0-neutral node; b) $\left(S, n_{0}\right)$ is an up-down node. In case a), it follows that there exists $S^{n, 0} \in \mathcal{S}_{\left(S, n_{0}\right)} \subset \mathcal{S}_{(S, n)}$ satisfying $\Delta_{n_{0}} S^{n, 0}=0$, hence

$$
-H_{n_{0}}\left(S^{n, 0}\right) \Delta_{n_{0}} S^{n, 0} \geq 0 \text {. }
$$

In case b), from the up down property, there exists $S^{n, 0} \in \mathcal{S}_{(S, n)}$ such that

$$
-H_{n_{0}}\left(S^{n, 0}\right) \Delta_{n_{0}} S^{n, 0} \geq \delta .
$$

If $N_{H}\left(S^{n, 0}\right) \leq n_{0}+1$, since $H_{i}(S)=0, \quad n \leq i<n_{0}$, then 4.10 or 4.11 show that $S^{*} \equiv S^{n, 0}$ satisfies the conditions of a contrarian trajectory we are looking for. So, assume $n_{0}+1<N_{H}\left(S^{n, 0}\right)$.

Proceeding recursively, we may assume that we have either constructed the desired trajectory or we have at our disposal a trajectory $S^{n, k} \in \mathcal{S}_{\left(S^{n, k-1}, n_{k}\right)}$, satisfying

$$
-H_{n_{k}}\left(S^{n, k}\right) \Delta_{n_{k}} S^{n, k} \geq 0 \quad \text { or } \quad-H_{n_{k}}\left(S^{n, k}\right) \Delta_{n_{k}} S^{n, k} \geq \delta
$$

as well as

$$
-H_{i}\left(S^{n, k}\right) \Delta_{i} S^{n, k}=0, \quad \text { for } n_{k-1}<i<n_{k} .
$$

We then look for the smallest $n_{k+1}$ satisfying $n_{k}<n_{k+1}<N_{H}\left(S^{n, k}\right)$ and $H_{n_{k+1}}\left(S^{n, k}\right) \neq 0$. If such $n_{k+1}$ does not exist the construction terminates by taking $S^{*} \equiv S^{n, k}$, thus concluding the proof. Otherwise, there exists $S^{n, k+1} \in \mathcal{S}_{\left(S^{n, k}, n_{k+1}\right)}$, and by means of the alternatives a) and b), and other considerations above, we obtain that the following holds:

$$
-H_{n_{k+1}}\left(S^{n, k+1}\right) \Delta_{n_{k+1}} S^{n, k+1} \geq 0 \quad \text { or } \quad-H_{n_{k+1}}\left(S^{n, k+1}\right) \Delta_{n_{k+1}} S^{n, k+1} \geq \delta,
$$

as well as

$$
-H_{i}\left(S^{n, k}\right) \Delta_{i} S^{n, k+1}=0, \quad \text { for } n_{k}<i<n_{k+1} .
$$

Continuing in this way, we have the following exclusive alternatives: i) we managed to construct the desired trajectory and, hence, the recursion terminates; ii) the recursion continues indefinitely, in which case we have:

$$
\begin{aligned}
-\sum_{k=0}^{m} H_{n_{k}}\left(S^{n, k}\right) \Delta_{n_{k}} S^{n, k} & =-\sum_{i=n}^{n_{m}} H_{i}\left(S^{n, m}\right) \Delta_{i} S^{n, m} \\
& \left.=-\sum_{i=n_{0}}^{n_{m}} H_{i}\left(S^{n, m}\right) \Delta_{i} S^{n, m} \geq[m+1-\hat{m})\right] \delta, \quad \forall m>\hat{m},
\end{aligned}
$$

where we used the fact that $H_{n_{k}}\left(S^{n, k}\right) \Delta_{n_{k}} S^{n, k}=H_{n_{k}}\left(S^{n, m}\right) \Delta_{n_{k}} S^{n, m}$ for $0 \leq k \leq$ $m$.

Let us show that 4.12 conflicts with 4.8 (recall that $H_{i}\left(S^{n, m}\right)=H_{i}(S)=0$, $\left.n \leq i<n_{0}\right)$ :

$$
\begin{aligned}
V_{0}+\sum_{i=0}^{n_{m}} H_{i}\left(S^{n, m}\right) \Delta_{i} S^{n, m} & =V_{0}+\sum_{i=0}^{n-1} H_{i}(S) \Delta_{i} S+\sum_{i=n}^{n_{m}} H_{i}\left(S^{n, m}\right) \Delta_{i} S^{n, m} \\
& \left.\leq V_{0}+\sum_{i=0}^{n-1} H_{i}(S) \Delta_{i} S-[m+1-\hat{m})\right] \delta<-A,
\end{aligned}
$$


where we obtained the last inequality by taking $m$ sufficiently large; let us denote the smallest integer satisfying (4.13) by $m^{*}$. This argument just proves that we can not have $n_{m^{*}} \leq N_{H}\left(S^{n, m^{*}}\right)$ as otherwise we have a contradiction with (4.8); it then follows that $n_{m^{*}}>N_{H}\left(S^{n, m^{*}}\right)>n$. To sum up: $-H_{i}\left(S^{n, m^{*}}\right) \Delta_{i} S^{n, m^{*}} \geq 0$ for all $n \leq i<N_{H}\left(S^{n, m^{*}}\right), S^{n, m^{*}} \in \mathcal{S}_{(S, n)}$ hence $S^{*} \equiv S^{n, m^{*}}$ is a contrarian trajectory that extends $S$ beyond $n$. The conditionally 0-neutral property then follows from Proposition 4

Remark 3. Notice that we have established more than is required in Definition 11 as each term in (4.4) has proven to be non-negative. The hypothesis 4.9 is only used to extract the required information from the up-down nodes.

The following corollary provides existence of the pricing interval in the setting of Theorem 2, we borrow all assumptions from that theorem but need to strengthen 4.9) so that the addition of portfolios obeys that equation as well.

Corollary 2. Consider a market $\mathcal{M}=\mathcal{S} \times \mathcal{H}$, a function $Z$ defined on $\mathcal{S}, S \in \mathcal{S}$, and $n \geq 0$ fixed. Assume that all hypotheses of Theorem 2 are satisfied, and that either $N_{H}$ is a stopping time for all $H \in \mathcal{H}$ or all $H \in \mathcal{H}$ are liquidated. Moreover, we strengthen (4.9) by assuming there are absolute positive constants $\delta_{H}>0, \rho>0$, $\gamma<0$ such that

$H_{i}\left(S^{\prime}\right) \in\left\{k \delta_{H}: k \in \mathbb{Z}\right\}$ and for all up-down nodes $\left(S^{\prime}, i\right), S^{\prime} \in \mathcal{S}_{(S, n)}, i \geq n$,

and the following holds:

$$
\sup _{\hat{S} \in \mathcal{S}_{\left(S^{\prime}, i\right)}} \Delta_{i} \hat{S}>\rho \quad \text { and } \quad \inf _{\hat{S} \in \mathcal{S}_{\left(S^{\prime}, i\right)}} \Delta_{i} \hat{S}<\gamma
$$

Then,

$$
\underline{V}_{n}(S, Z, \mathcal{M}) \leq \bar{V}_{n}(S, Z, \mathcal{M}) .
$$

Proof. Let $\widetilde{\mathcal{H}} \equiv \mathcal{H}+\mathcal{H}$. We will argue that Theorem 2 is applicable to $\mathcal{S} \times \widetilde{\mathcal{H}}$ and borrow the notation used in that theorem. Assumption (2) in Theorem 2 can be made to hold for $\widetilde{\mathcal{H}}$ by defininig $A\left(H^{1}+H^{2}\right)=A\left(H^{1}\right)+A\left(H^{2}\right)$ whenever $H^{k} \in \mathcal{H}$, $k=1,2$. Also assumption (3) in Theorem 2 holds with $\delta \equiv \min \left(\delta_{H} \rho,-\gamma \delta_{H}\right)$ for $\widetilde{\mathcal{H}}$ given our assumption 4.14). Therefore, $\mathcal{M}=\mathcal{S} \times \widetilde{\mathcal{H}}$ is conditionally 0-neutral at $(S, n)$. It follows that

$$
\underline{V}_{n}(S, Z, \mathcal{M}) \leq \underline{V}_{n}(S, Z, \widetilde{\mathcal{M}}) \leq \bar{V}_{n}(S, Z, \widetilde{\mathcal{M}}) \leq \bar{V}_{n}(S, Z, \mathcal{M}),
$$

where the innermost inequality follows from Theorem 1

4.2. Relation with arbitrage. This short section connects the approach of the paper with the usual notion of arbitrage. In particular, Corollary 4 provides a sufficient condition for a market to be arbitrage free.

See Equation 2.2 for the precise definitions of quantities appearing below.

Definition 12 (Arbitrage-Free Market). Given a market $\mathcal{M}, H \in \mathcal{H}$ is an arbitrage strategy if: 
- $\forall S \in \mathcal{S}, V_{H}\left(N_{H}(S), S\right) \geq V_{H}\left(0, S_{0}\right)$.

- $\exists S^{*} \in \mathcal{S}$ satisfying $\left.V_{H}\left(N_{H}\left(S^{*}\right), S^{*}\right)\right)>V_{H}\left(0, S_{0}\right)$.

We will say that $\mathcal{M}$ is arbitrage-free if $\mathcal{H}$ contains no arbitrage strategies.

The above definition is the classical probabilistic definition ([4]), but inequalities are now required to hold pointwise (as opposed to a.e.). When $N_{H}(S)=$ $T, \forall S, \forall H$, it corresponds to the notion of 1-point arbitrage introduced in 20] and [6]. Comparing with Definition 6. $H$ with initial capital $V_{0}=0$ is an arbitrage strategy if and only if it is an extended arbitrage with respect to $Z=0$.

The following proposition mimics Proposition 4 s some of the similarity is lost because we have not introduced conditional versions of arbitrage.

Proposition 5. $\mathcal{M}$ is arbitrage-free if and only if for each $H \in \mathcal{H}$ we have:

$\exists S^{0} \in \mathcal{S}$ such that $H$ and $S^{0}$ are 0 -contrarian beyond $n=0$, or

$$
\sum_{i=0}^{N_{H}(S)-1} H_{i}(S) \Delta_{i} S=0 \quad \forall S \in \mathcal{S} .
$$

Clearly if $H$ and $S$ are $\epsilon^{\prime}$-contrarian then they will be also $\epsilon$-contrarian if $\epsilon \geq \epsilon^{\prime}$; this comment and Proposition 4 imply the following corollary, which shows that 0 -neutral is a necessary condition for a market to be arbitrage-free.

Corollary 3. If $\mathcal{M}$ is arbitrage-free, then $\mathcal{M}$ is 0-neutral.

The converse of Corollary 3 does not hold in general. Consider a market with $N_{H}=1, \forall H \in \mathcal{H}$. If $\sup _{S \in \mathcal{S}} \Delta_{0} S>0$ and $\inf _{S \in \mathcal{S}} \Delta_{0} S=0$, it provides a clear arbitrage with $H_{0} \equiv 1$, nonetheless the market is 0 -neutral. We have seen in the previous section that a well defined option pricing methodology is still possible.

A study of the proof of Theorem 2 in conjunction with Proposition 5 gives the following corollary.

Corollary 4. Assume the same hypothesis as in Theorem 2 and, furthermore, require $\hat{m}=0$. Then, $\mathcal{M}=\mathcal{S} \times \mathcal{H}$ is arbitrage-free.

\section{Attainability. Formal martingale properties}

This section concerns the notion of attainability, as well as a generalization of this notion and some implications. Under the assumption of attainability the minmax bounds are additive; we also present results providing formal analogues of martingale properties.

Definition 13. Given a market $\mathcal{M}=\mathcal{S} \times \mathcal{H}$ and non-negative numbers $\epsilon^{\uparrow}$ and $\epsilon^{\downarrow}$, a function $Z$ is called $\epsilon^{\uparrow}$-upward attainable if there exists $H^{\uparrow} \in \mathcal{H}$ and a number $V^{\uparrow}$ such that

$$
0 \leq V^{\uparrow}+\sum_{i=0}^{N_{H \uparrow}(S)-1} H_{i}^{\uparrow}(S) \Delta_{i} S-Z(S) \leq \epsilon^{\uparrow} \quad \forall S \in \mathcal{S} .
$$


Analogously, $Z$ is called $\epsilon^{\downarrow}$-downward attainable if there exists $H^{\downarrow} \in \mathcal{H}$ and a number $V^{\downarrow}$ such that

$$
0 \leq-V^{\downarrow}-\sum_{i=0}^{N_{H \downarrow}(S)-1} H_{i}^{\downarrow}(S) \Delta_{i} S+Z(S) \leq \epsilon^{\downarrow} \quad \forall S \in \mathcal{S} .
$$

Finally $Z$ is called attainable (or replicable) if it is 0-upward (or downward) attainable, in such a case we use the notation $H^{z}=H^{\uparrow}$ and $V_{H^{z}}=V^{\uparrow}$.

The next proposition shows that the distance separating the price bounds is bounded by the maximum profits.

Proposition 6. Let $\mathcal{M}=\mathcal{S} \times \mathcal{H}$ be a market, $S^{*} \in \mathcal{S}, k \geq 0$ and $Z$ a function on $\mathcal{S}$. Assume all $H \in \mathcal{H}$ are liquidated and consider the statements:

a) $Z$ is $\epsilon^{\uparrow}$-upward attainable and $-H^{\uparrow} \in \mathcal{H}$.

b) $Z$ is $\epsilon^{\downarrow}$-downward attainable and $-H^{\downarrow} \in \mathcal{H}$.

Then, the following holds:

$$
\bar{V}_{k}\left(S^{*}, Z, \mathcal{M}\right)-\underline{V}_{k}\left(S^{*}, Z, \mathcal{M}\right) \leq \epsilon,
$$

where $\epsilon=\epsilon^{\uparrow}$ if a) holds, $\epsilon=\epsilon^{\downarrow}$ if b) holds and $\epsilon=\epsilon^{\uparrow} \wedge \epsilon^{\downarrow}$ if a) and b) hold.

Proof. Introduce the notation $V^{\uparrow}\left(k, S^{*}\right) \equiv V^{\uparrow}+\sum_{i=0}^{k-1} H_{i}^{\uparrow}\left(S^{*}\right) \Delta_{i} S^{*}$ and $V^{\downarrow}\left(k, S^{*}\right) \equiv$ $V^{\downarrow}+\sum_{i=0}^{k-1} H_{i}^{\downarrow}\left(S^{*}\right) \Delta_{i} S^{*}$. If a) holds, it follows from (5.1) and $-H^{\uparrow} \in \mathcal{H}$ that

$$
-\underline{V}_{k}\left(S^{*}, Z, \mathcal{M}\right) \leq \sup _{S \in \mathcal{S}_{\left(S^{*}, k\right)}}\left[-Z(S)-\sum_{i=k}^{N_{H \uparrow}(S)-1}-H_{i}^{\uparrow}(S) \Delta_{i} S\right]=-V^{\uparrow}\left(k, S^{*}\right)+\epsilon^{\uparrow} .
$$

Display (5.1) also implies $\bar{V}_{k}\left(S^{*}, Z, \mathcal{M}\right) \leq V^{\uparrow}\left(k, S^{*}\right)$, therefore

$$
\underline{V}_{k}\left(S^{*}, Z, \mathcal{M}\right) \geq V^{\uparrow}\left(k, S^{*}\right)-\epsilon^{\uparrow},
$$

so $(5.3)$ holds.

Similarly if b) holds, it follows from 5.2 that

$$
\bar{V}_{k}\left(S^{*}, Z, \mathcal{M}\right) \leq V^{\downarrow}\left(k, S^{*}\right)+\epsilon^{\downarrow} \quad \text { and } \quad \underline{V}_{k}\left(S^{*}, Z, \mathcal{M}\right) \geq V^{\downarrow}\left(k, S^{*}\right) .
$$

So (5.3) holds.

In general, the bounds are not linear as functions of the payoff. The following result presents a case where the bounds are additive. For the sake of generality the corollary below directly assumes $\underline{V}_{k}\left(S^{*}, Z, \mathcal{M}\right) \leq \bar{V}_{k}\left(S^{*}, Z, \mathcal{M}\right)$ instead of any specific hypothesis leading to this inequality.

Corollary 5. Consider a market $\mathcal{M}=\mathcal{S} \times \mathcal{H}, S^{*} \in \mathcal{S}, k \geq 0$ and $Z$ a function on $\mathcal{S}$. Assume all $H \in \mathcal{H}$ are liquidated and $\underline{V}_{k}\left(S^{*}, Z, \mathcal{M}\right) \leq \bar{V}_{k}\left(S^{*}, Z, \mathcal{M}\right)$.

a) If $Z$ is attainable with portfolio $H^{z}$ and $-H^{z} \in \mathcal{H}$ then

$$
\bar{V}_{k}\left(S^{*}, Z, \mathcal{M}\right)=\underline{V}_{k}\left(S^{*}, Z, \mathcal{M}\right)=V_{H^{z}}+\sum_{i=0}^{k-1} H_{i}^{z}\left(S^{*}\right) \Delta_{i} S^{*} .
$$


b) If $Z_{j}, j=1,2$, are attainable with portfolios $H^{z_{j}}$ satisfying: $-H^{z_{j}} \in \mathcal{H}$ and $H^{z_{1}}+H^{z_{2}} \in \mathcal{H}$, then

$$
\bar{V}_{k}\left(S^{*}, Z_{1}+Z_{2}, \mathcal{M}\right)=\bar{V}_{k}\left(S^{*}, Z_{1}, \mathcal{M}\right)+\bar{V}_{k}\left(S^{*}, Z_{2}, \mathcal{M}\right)
$$

Proof. By assumption,

$$
Z(S)=V_{H^{z}}(k, S)+\sum_{i=k}^{N_{H^{z}}(S)-1} H_{i}^{z}(S) \Delta_{i} S, \quad \forall S \in \mathcal{S},
$$

where we have used the abbreviation $V_{H^{z}}(k, S) \equiv V_{H^{z}}+\sum_{i-0}^{k-1} H_{i}^{z}(S) \Delta_{i} S$, with some abuse of notation (as $V_{H^{z}}$ may not be necessarily equal to $V_{H^{z}}(0, S)$ ). It then follows that

$$
\bar{V}_{k}\left(S^{*}, Z, \mathcal{M}\right) \leq V_{H^{z}}\left(k, S^{*}\right) .
$$

Similarly, since $-H^{z} \in \mathcal{H}$, thus

$$
\underline{V}_{k}\left(S^{*}, Z, \mathcal{M}\right)=-\bar{V}_{k}\left(S^{*},-Z, \mathcal{M}\right) \geq V_{H^{z}}\left(k, S^{*}\right) .
$$

Notice that Proposition 6 is applicable and (5.3), together with our hypothesis, gives $\underline{V}_{k}\left(S^{*}, Z, \mathcal{M}\right)=\bar{V}_{k}\left(S^{*}, Z, \mathcal{M}\right)$. This equality combined with the above inequalities concludes the proof of (5.4).

The proof of (5.5) follows from (5.4) after noticing that $Z \equiv Z_{1}+Z_{2}$ is attainable and $V_{H^{z}}\left(k, S^{*}\right)=V_{H^{z_{1}}}\left(k, S^{*}\right)+V_{H^{z_{2}}}\left(k, S^{*}\right)$.

The following corollary expresses a consistency result, namely today's stock price is the minmax price in a 0-neutral market $\mathcal{M}$. Assumptions leading to the conclusion $\underline{V}(S, Z, \mathcal{M}) \leq \bar{V}(S, Z, \mathcal{M})$, for any $S \in \mathcal{S}$ (as in Theorem 1, Proposition 2, and Corollary 2), will be required. The corollary below can be considered as a trajectorial analogue of the optional stopping theorem for martingales, one could also prove that a stopped 0-neutral trajectory set is also 0-neutral and so providing a fuller analogy to the mentioned theorem.

For a stopping time $\tau$ we introduce notation to be used in the following corollary. Define $G^{\tau}$ by:

$$
G_{i}^{\tau}(S)=1 \quad \text { for } 0 \leq i \leq \tau(S)-1, \quad N_{G^{\tau}}(S)=\tau(S), \quad \text { and } \quad V_{G^{\tau}}\left(0, S_{0}\right)=S_{0} ;
$$

also set $G_{i}^{\tau}(S)=0$ for $i \geq \tau(S)$.

Corollary 6. Let $\tau$ be a stopping time and $\mathcal{M}=\mathcal{S} \times \mathcal{H}$ a market and assume all $H \in \mathcal{H}$ are liquidated. Fix $S^{*} \in \mathcal{S}, k \geq 0$ and assume the conditions on $\mathcal{M}$ that assure the existence of a pricing interval. If $G^{\tau}$ and $-G^{\tau}$ belong to $\mathcal{H}$, then

$$
\underline{V}_{k}\left(S^{*}, S_{\tau}, \mathcal{M}\right)=\bar{V}_{k}\left(S^{*}, S_{\tau}, \mathcal{M}\right)=S_{k \wedge \tau\left(S^{*}\right)}^{*},
$$

where $S_{\tau}$ denotes the function $Z$, defined on $\mathcal{S}$ by $Z(S)=S_{\tau(S)}$.

Conversely, set $\tau(S) \equiv k+1$ for all $S$, and assume that for all $H \in \mathcal{H}, H+G^{\tau} \in$ $\mathcal{H}$. Then if $\bar{V}_{k}\left(S^{*}, S_{\tau}, \mathcal{M}\right)=S_{k}^{*}$, it follows that $\mathcal{M}$ is conditionally 0-neutral at $\left(S^{*}, k\right)$. 
Proof. Notice that $S_{\tau(S)}=S_{0}+\sum_{i=0}^{\tau(S)-1} \Delta_{i} S$, for any $S \in \mathcal{S}$, and $G^{\tau}$ is clearly non-anticipative. So $Z=S_{\tau}$ is attainable. Therefore, Corollary 5 is applicable giving 5.6 since $V_{G^{\tau}}\left(k, S^{*}\right)=S_{k \wedge \tau\left(S^{*}\right)}^{*}$.

For the second statement, if $\bar{V}_{k}\left(S^{*}, S_{\tau}, \mathcal{M}\right)=S_{k}^{*}$, then

$$
\begin{aligned}
0 & \left.=\inf _{H \in \mathcal{H}}\left\{\sup _{S \in \mathcal{S}_{\left(S^{*}, k\right)}}\left[S_{\tau(S)}-S_{k}-\sum_{i=k}^{N_{H}(S)-1} H_{i}(S) \Delta_{i} S\right)\right]\right\} \\
& \left.=\inf _{H \in \mathcal{H}}\left\{\sup _{S \in \mathcal{S}_{\left(S^{*}, k\right)}}\left[-\left(\sum_{i=k}^{N_{H}(S)-1} H_{i}(S)-\sum_{i=k}^{\tau(S)-1} G_{i}^{\tau}(S)\right) \Delta_{i} S\right)\right]\right\} \\
& \left.\leq \inf _{H \in \mathcal{H}}\left\{\sup _{S \in \mathcal{S}_{\left(S^{*}, k\right)}}\left[-\sum_{i=k}^{N_{H}(S)-1} H_{i}(S) \Delta_{i} S\right)\right]\right\} .
\end{aligned}
$$

The last inequality holds, because $H+G^{\tau} \in \mathcal{H}$ for all $H \in \mathcal{H}$, and then $\mathcal{H} \subset\{H-$ $\left.G^{\tau}: H \in \mathcal{H}\right\}$. Finally, since the portfolio $0 \in \mathcal{H}$, it follows that $\bar{V}_{k}\left(S^{*}, 0, \mathcal{M}\right)=0$. Therefore, $\mathcal{M}$ is conditionally 0 -neutral at $\left(S^{*}, k\right)$.

\section{RELation to RISK NEUtral PRICing}

This section defines a market $\mathcal{M}$ from a continuous-time martingale market. The results give some perspective to our approach and allow to establish connections between the minmax bounds and risk neutral pricing. Trajectory spaces are defined by stopping times samples of continuous-time martingale paths. A main point to emphasize is that the 0-neutral property holds due to the discrete sampling via stopping times and the martingale property; we do make use of the extended formalism as described in Section 2.2

Consider a stochastic market model consisting of a probability space $(\Omega, \mathcal{F}, P)$ where $\mathcal{F}=\left\{\mathcal{F}_{t}\right\}_{0 \leq t \leq T}$ is a continuous-time filtration. Also there is an adapted process $X=\left\{X_{t}\right\}_{0 \leq t \leq T}$ taking values on $\mathbb{R}$, we also assume $\mathcal{F}_{0}$ is the trivial sigma algebra. Moreover, there exists a measure $Q$, equivalent to $P$, such that $X$ is a martingale relative to $\mathcal{F}$ and $Q$. This setting represents an arbitrage-free (in a stochastic sense), 1-dimensional market with a deterministic bank account with 0 interest rates. A European payoff $Y$ is a real valued function defined on $\Omega$, nonnegative, $\mathcal{F}_{T}$-measurable with respect to $Q$. A risk neutral price of such a claim is then given by $\mathbb{E}_{Q}(Y)$, where the expectation is with respect to a measure $Q$.

Naturally, we assume that quantities defined on $\Omega$ are only defined a.e., we will not explicitly indicate this fact in every instance but will do so in critical aspects of the constructions. The context should make it easy to realize if we are referring to filtration-based stopping times or trajectory-based stopping times.

6.1. Martingale trajectory market. A sequence of (filtration-based) stopping times $\tau=\left\{\tau_{i}\right\}$, relative to the filtration $\mathcal{F}$, is said to be admissible if $\tau_{i} \leq \tau_{i+1}$, $0=\tau_{0}$ and, for a given $\omega$, there exists a smallest integer $M=M_{\tau}(\omega)$ such that $\tau_{M}(\omega)=T$. All sequences of stopping times considered in the remaining of this 
section are admissible, this fact may not be explicitly indicated. For simplicity, we may write $X_{\tau_{i}(\omega)}(\omega)$, and related quantities, as $X_{\tau_{i}}(\omega)$.

On the stochastic side, at some points we will look at portfolios of the form

$$
u_{0}^{y}+\sum_{i=0}^{M_{\tau}-1} U_{i}^{y}\left(X_{\tau_{i+1}}-X_{\tau_{i}}\right), \quad \text { for a constant } u_{0}^{y},
$$

where the investment $U_{i}^{y}$ is $\mathcal{F}_{\tau_{i}}$-measurable. For technical reasons we will assume there exists a countable subset $C$ of $[0, T]$, with $0, T \in C$, and the quantities $U_{i}(\omega)$ depend only on $X_{s}, s \in C$. This assumption is formalized next.

We will assume that all $\tau=\left\{\tau_{i}\right\}$ are such that the $\tau_{i}$ take values on $C$. Let $\Omega_{0}$ be a set of full measure where all random variables $X_{s}, s \in C$, are defined and let $\Omega_{0}(\tau)$ be a set of full measure contained in $\Omega_{0}$ where all random variables $\left\{X_{\tau_{i}}\right\}$ are defined.

For given $\tau$ and $\omega \in \Omega_{0}(\tau)$ define $x_{\omega, \tau_{i}}: C \rightarrow \mathbb{R}$ by $x_{\omega, \tau_{i}}(s)=X_{s \wedge \tau_{i}(\omega)}(\omega)$; also set

$$
\mathcal{U}(\tau)=\left\{U=\left\{U_{i}\right\}_{i \geq 0}: U_{i}: \Omega_{0}(\tau) \rightarrow \mathbb{R}, U_{i}(\omega)=\mathbf{1}_{\left\{M_{\tau}>i\right\}}(\omega) F_{i}^{U}\left(x_{\omega, \tau_{i}}\right)\right\},
$$

where $F_{i}^{U}: \mathbb{R}^{C} \rightarrow \mathbb{R}$ is a bounded and Borel measurable function.

Recall that the Borel subsets of $\mathbb{R}^{C}$, denoted by $\mathcal{B}\left(\mathbb{R}^{C}\right)$, are generated by the family of cylinders $\left\{x \in \mathbb{R}^{C}: x\left(c_{j}\right) \in \Gamma, 1 \leq j \leq n\right\}$, with $\Gamma \in \mathcal{B}(\mathbb{R})$.

Corollary 7. Let $U=\left\{U_{i}\right\}_{i \geq 0} \in \mathcal{U}(\tau)$. Assume $M_{\tau}$ is a $\mathcal{F}_{\tau} \equiv\left\{\mathcal{F}_{\tau_{i}}\right\}_{i \geq 0}$ stopping time. Then $U_{i} \in \mathcal{F}_{\tau_{i}}$ for all $i \geq 0$.

Proof. Fix $i \geq 0$. Consider the function $\phi:\left(\Omega, \mathcal{F}_{\tau_{i}}\right) \rightarrow\left(\mathbb{R}^{C}, \mathcal{B}\left(\mathbb{R}^{C}\right)\right)$, defined by $\phi(\omega)=x_{\omega, \tau_{i}}$. Lemma 3 in Appendix E shows that $\phi$ is measurable. It follows that for $F_{i}$ as in $(6.1)$ and any $\Gamma \in \mathcal{B}(\mathbb{R})$

$$
\left(F_{i} \circ \phi\right)^{-1}(\Gamma)=\phi^{-1}\left(F_{i}^{-1}(\Gamma)\right) \in \mathcal{F}_{\tau_{i}},
$$

since $F_{i}^{-1}(\Gamma) \in \mathcal{B}\left(\mathbb{R}^{C}\right)$, thus $F_{i} \circ \phi$ is $\mathcal{F}_{\tau_{i}}$-measurable.

Given that $U_{i}=\mathbf{1}_{\left\{M_{\tau}>i\right\}}\left(F_{i} \circ \phi\right)$, and $\mathbf{1}_{\left\{M_{\tau}>i\right\}}$ is $\mathcal{F}_{\tau_{i}}$-measurable, $U_{i} \in \mathcal{F}_{\tau_{i}}$ too.

Regarding the trajectories defined below: we sample, a finite (but arbitrary) number of times, every random trajectory, and we record those values as well as other information that will be needed (see comments below). The notation below is from Section 2.2.

Given $\tau$, define

$$
\begin{aligned}
\mathcal{S}^{\mathcal{W}}(\tau)=\left\{\mathbf{S}=(S, W)=\left\{\left(S_{i}, W_{i}\right)\right\}_{i \geq 0}\right. & : \exists \omega \in \Omega_{0}(\tau), \\
S_{i} & \left.=X_{\tau_{i}(\omega)}(\omega), W_{i}=\left(\tau_{i}(\omega), x_{\omega, \tau_{i}}\right)\right\} .
\end{aligned}
$$

Also define $\mathcal{S}^{\mathcal{W}} \equiv \cup_{\tau} \mathcal{S}^{\mathcal{W}}(\tau)$, where the union is taken over admissible sequences of stopping times.

The inclusion of $\tau_{i}(\omega)$ and $x_{\omega, \tau_{i}}$ in $W_{i}$ allows the functions $H_{i}$ and $N_{H}$ (defined below) to be well defined and $H$ to be non-anticipative. Equality is defined as 
follows. Let $(S, W) \in \mathcal{S}^{\mathcal{W}}(\tau),\left(S^{\prime}, W^{\prime}\right) \in \mathcal{S}^{\mathcal{W}}\left(\tau^{\prime}\right)$; then $(S, W)=\left(S^{\prime}, W^{\prime}\right)$ if and only if $X_{\tau_{i}}(\omega)=X_{\tau_{i}^{\prime}}\left(\omega^{\prime}\right), \tau_{i}(\omega)=\tau_{i}^{\prime}\left(\omega^{\prime}\right)$, and $x_{\omega, \tau_{i}}=x_{\omega^{\prime}, \tau_{i}^{\prime}}, \quad \forall i \geq 0$.

As a shorthand notation, the association between martingale paths, the stopping time and the trajectory values described in 6.2 will be denoted by $\mathbf{S} \leftrightarrows X_{\tau}(\omega)$.

Define

$$
\mathcal{H}=\left\{H=\left\{H_{i}\right\}_{i \geq 0}: H_{i}: \mathcal{S}^{\mathcal{W}} \rightarrow \mathbb{R}\right\},
$$

where the functions $H_{i}$ are defined as follows: there exists a bounded Borel function $F_{i}: \mathbb{R}^{C} \rightarrow \mathbb{R}$ with the property that, for $\mathbf{S} \leftrightarrows X_{\tau}(\omega)$

$$
H_{i}(\mathbf{S})=\mathbf{1}_{\left\{M_{\tau}>i\right\}}(\omega) F_{i}\left(x_{\omega, \tau_{i}}\right) \quad \text { and } \quad N_{H}(\mathbf{S})=M_{\tau}(\omega) .
$$

Proposition 7 below shows that $H$ and $N_{H}$ are well defined and $H$ is non-anticipative. We allow arbitrary values for $V_{H}\left(0, S_{0}\right)$, initial portfolio values, and define the bank account value sequence $\left\{B_{i}\right\}$ such that portfolios are self financing as indicated in Remark 1

The association described in (6.4) will be denoted by $H \leftrightarrows F$.

Proposition 7. Portfolios $H$ and functions $N_{H}$ introduced by (6.3) and (6.4) are well defined on $\mathcal{S}^{\mathcal{W}}$ and portfolios $H$ are non-anticipative as well.

Proof. Let $\mathbf{S}=(S, W), \mathbf{S}^{\prime}=\left(S^{\prime}, W^{\prime}\right) \in \mathcal{S}^{\mathcal{W}}$ with $\mathbf{S} \leftrightarrows X_{\tau}(\omega)$ and $\mathbf{S}^{\prime} \leftrightarrows X_{\tau^{\prime}}\left(\omega^{\prime}\right)$

1. Assume that $\mathbf{S}=\mathbf{S}^{\prime}$, then $W=W^{\prime}$ implies $\tau_{j}^{\prime}\left(\omega^{\prime}\right)=\tau_{j}(\omega) \forall j \geq 0$. Thus

$$
\tau_{j}^{\prime}\left(\omega^{\prime}\right)=\tau_{j}(\omega)<T \text { for } 0 \leq j<M_{\tau}(\omega) \text { so } \quad M_{\tau^{\prime}}\left(\omega^{\prime}\right) \geq M_{\tau}(\omega) .
$$

Since the former reasoning is symmetric, $M_{\tau^{\prime}}\left(\omega^{\prime}\right)=M_{\tau}(\omega)$, and $N_{H}$ is well defined.

Fix now $i \geq 0$, therefore by the previous statement, $M_{\tau}(\omega)>i$ if and only if $M_{\tau^{\prime}}\left(\omega^{\prime}\right)>i$. Moreover, since $x_{\omega, \tau_{i}}=x_{\omega^{\prime}, \tau_{i}^{\prime}}$, it follows that

$$
H_{i}\left(\mathbf{S}^{\prime}\right)=\mathbf{1}_{\left\{M_{\tau^{\prime}}>i\right\}}\left(\omega^{\prime}\right) F_{i}\left(x_{\omega^{\prime}, \tau_{i}^{\prime}}\right)=\mathbf{1}_{\left\{M_{\tau}>i\right\}}(\omega) F_{i}\left(x_{\omega, \tau_{i}}\right)=H_{i}(\mathbf{S}) .
$$

This shows that $H$ is well defined.

2. To prove that $H$ is non-anticipative, let $i \geq 0$ fixed and assume $\left(S_{k}, W_{k}\right)=$ $\left(S_{k}^{\prime}, W_{k}^{\prime}\right), k=0, \ldots, i$. We need to prove that $H_{i}(\mathbf{S})=H_{i}\left(\mathbf{S}^{\prime}\right)$. Observe that $M_{\tau}(\omega) \leq i$ if and only if $M_{\tau^{\prime}}\left(\omega^{\prime}\right) \leq i$. Indeed, if $M_{\tau}(\omega)=N \leq i$, then $\tau_{N}^{\prime}\left(\omega^{\prime}\right)=$ $\tau_{N}(\omega)=T$, so $M_{\tau^{\prime}}\left(\omega^{\prime}\right) \leq N \leq i$ and, consequently, $H_{i}(\mathbf{S})=H_{i}\left(\mathbf{S}^{\prime}\right)$ if that is the case.

On the other hand, since $x_{\omega, \tau_{k}}=x_{\omega^{\prime}, \tau_{k}^{\prime}}$ for $0 \leq k \leq i$, again

$$
H_{i}\left(\mathbf{S}^{\prime}\right)=\mathbf{1}_{\left\{M_{\tau^{\prime}}>i\right\}}\left(\omega^{\prime}\right) F_{i}\left(x_{\omega^{\prime}, \tau_{i}^{\prime}}\right)=\mathbf{1}_{\left\{M_{\tau}>i\right\}}(\omega) F_{i}\left(x_{\omega, \tau_{i}}\right)=H_{i}(\mathbf{S}) .
$$

Define the martingale trajectory markets

$$
\mathcal{M}=\mathcal{S}^{\mathcal{W}} \times \mathcal{H} \text { and } \mathcal{M}(\tau)=\mathcal{S}^{\mathcal{W}}(\tau) \times \mathcal{H}
$$

where, in the case of $\mathcal{M}(\tau)$, portfolios $H$ act on $\mathcal{S}^{\mathcal{W}}(\tau) \subseteq \mathcal{S}^{\mathcal{W}}$ by restriction.

Remark 4. To lighten the notation, we will write $\mathcal{S}_{(\mathbf{S}, k)}^{\mathcal{W}}(\tau)$ as $\mathcal{S}_{(\mathbf{S}, k)}(\tau)$. Similarly, we will write $\mathcal{S}_{(\mathbf{S}, k)}^{\mathcal{W}}$ as $\mathcal{S}_{(\mathbf{S}, k)}$ 
As preparation for the next result, let $\mathcal{P}=\mathcal{P}(P)$ be the set of all martingale probability measures equivalent to $P$ and let $\mathbb{E}_{Q}(Y)$ denote expectation with respect to the probability measure $Q$.

For the next two results in this section, we are going to assume conditions under which $\left\{X_{\tau_{i}}\right\}_{i \geq 0}$ behaves as a martingale with respect to $\mathcal{F}_{\tau} \equiv\left\{\mathcal{F}_{\tau_{i}}\right\}_{i \geq 0}$; namely,

$$
\mathbb{E}_{Q}\left[X_{\tau_{i}} \mid \mathcal{F}_{\tau_{k}}\right]=X_{\tau_{k}}, \quad i \geq 1, k \leq i .
$$

Proposition 8. Let $Y$ be a European payoff and, for a given $\tau$, define $Z_{\tau}(\mathbf{S})=$ $Y(\omega)$ where $S \leftrightarrows X_{\tau}(\omega)$ and $\omega \in \Omega_{0}(\tau)$. Assume that

$$
\sup _{\mathbf{S} \in \mathcal{S}^{\mathcal{W}}(\tau)}\left[Z_{\tau}(\mathbf{S})-\sum_{i=0}^{N_{H^{*}}(\mathbf{S})-1} H_{i}^{*}(\mathbf{S}) \Delta_{i} S\right]<\infty,
$$

for some $H^{*} \in \mathcal{H}$. Assume also that $M_{\tau}$ is a stopping time w.r.t. $\mathcal{F}_{\tau}$, and that the hypotheses of Lemma 4, in Appendix E, are satisfied. Then,

$$
\begin{aligned}
\sup _{\tau} \underline{V}\left(\left(S_{0}, W_{0}\right), Z_{\tau}, \mathcal{M}(\tau)\right) & \leq \inf _{Q \in \mathcal{P}} \mathbb{E}_{Q}(Y) \leq \sup _{Q \in \mathcal{P}} \mathbb{E}_{Q}(Y) \\
& \leq \inf _{\tau} \bar{V}\left(\left(S_{0}, W_{0}\right), Z_{\tau}, \mathcal{M}(\tau)\right) .
\end{aligned}
$$

Proof. Notice that $\Omega_{0}(\tau)$, and hence also $\mathcal{S}^{\mathcal{W}}(\tau)$, depends on $P$ only through null sets of $P$; therefore, it remains unchanged if defined through any $Q \in \mathcal{P}$.

For $i \geq 1$ set $\Omega_{i}(\tau) \equiv\left\{\omega \in \Omega_{0}(\tau): M_{\tau}(\omega)>i\right\}$; it is clear that $\Omega_{i}(\tau) \in \mathcal{F}_{\tau_{i}}$, and if $M_{\tau}$ is bounded then $\Omega_{0}(\tau)=\Omega_{m}^{c}(\tau)$ for some $m \geq 1$. Consider $H \in \mathcal{H}$ and $\mathbf{S}=(S, W) \in \mathcal{S}^{\mathcal{W}}$ with $S \leftrightarrows X_{\tau}(\omega)$. For any $i \geq 0$, from definition (6.3), $H_{i}(\mathbf{S})=\mathbf{1}_{\Omega_{i}(\tau)}(\omega) F_{i}\left(x_{\omega, \tau_{i}}\right)$, with $F_{i}: \mathbb{R}^{C} \rightarrow \mathbb{R}$ a bounded Borel function. Defining $U_{i}(\omega)=\mathbf{1}_{\Omega_{i}(\tau)}(\omega) F_{i}\left(x_{\omega, \tau_{i}}\right)$, it follows that $U=\left\{U_{i}\right\}_{i \geq 0} \in \mathcal{U}(\tau)$, and by Corollary 7 $U_{i} \in \mathcal{F}_{\tau_{i}}$. We have

$$
\begin{array}{r}
Y(\omega)-\sum_{i=0}^{M_{\tau}(\omega)-1} U_{i}(\omega)\left(X_{\tau_{i+1}}(\omega)-X_{\tau_{i}}(\omega)\right)=Z_{\tau}(\mathbf{S})-\sum_{i=0}^{N_{H}(\mathbf{S})-1} H_{i}(\mathbf{S}) \Delta_{i} S \\
\leq \sup _{\mathbf{S} \in \mathcal{S}^{\mathcal{W}}(\tau)}\left[Z_{\tau}(\mathbf{S})-\sum_{i=0}^{N_{H}(\mathbf{S})-1} H_{i}(\mathbf{S}) \Delta_{i} S\right]
\end{array}
$$

So, since it holds with $H^{*}$, and $\sum_{i=0}^{M_{\tau}-1} U_{i}\left(X_{\tau_{i+1}}-X_{\tau_{i}}\right)$ is integrable by Lemma 4 $Y$ also results integrable.

Taking infimum and supremum on $\mathcal{S}^{\mathcal{W}}$ in 6.6 , and then expectation w.r.t. $Q \in \mathcal{P}$,

$$
\begin{array}{r}
-\sup _{\mathbf{S} \in \mathcal{S}^{\mathcal{W}}(\tau)}\left[-Z_{\tau}(\mathbf{S})-\sum_{i=0}^{N_{H}(\mathbf{S})-1}-H_{i}(\mathbf{S}) \Delta_{i} S\right] \leq \mathbb{E}_{Q}\left[Y-\sum_{i=0}^{M_{\tau}-1} U_{i}\left(X_{\tau_{i+1}}-X_{\tau_{i}}\right)\right] \\
=\mathbb{E}_{Q}[Y] \leq \sup _{\mathbf{S} \in \mathcal{S}^{\mathcal{W}}(\tau)}\left[Z_{\tau}(\mathbf{S})-\sum_{i=0}^{N_{H}(\mathbf{S})-1} H_{i}(\mathbf{S}) \Delta_{i} S\right], \quad
\end{array}
$$


where we have used the fact that $\left\{X_{\tau_{i}}\right\}_{i \geq 0}$ is a martingale [17, Thm. 1.86] and Lemma 4

Notice that $-\mathcal{H}=\mathcal{H}$, we then obtain 6.5 by taking supremum and infimum over $H$ in the left hand side and right hand side of (6.7) respectively and then making use of the fact that $\tau$, admissible, was taken arbitrary in the above arguments.

Remark 5. The condition $\sup _{\mathbf{S} \in \mathcal{S}^{\mathcal{W}}(\tau)}\left[Z_{\tau}(\mathbf{S})-\sum_{i=0}^{N_{H}(\mathbf{S})-1} H_{i}(\mathbf{S}) \Delta_{i} S\right]<\infty$ required in the previous Proposition, is equivalent to $\bar{V}\left(S_{0}, Z_{\tau}, \mathcal{M}_{\tau}\right)<\infty$, so it is satisfied when $Z_{\tau}$ is an upper minmax function (see [13]).

For the case when $\Omega$ is finite or, more generally, purely atomic, it should be clear that all nodes in $\mathcal{S}^{\mathcal{W}}$ are arbitrage-free nodes according to Definition 10 (this can be readily obtained from Theorem 3.1 in [22]).

The next result represents the key property connecting martingale trajectory markets with the formalism of the paper.

Theorem 3. Consider a martingale trajectory market $\mathcal{M}(\tau)$ which satisfies the conditions of Lemma 4 in Appendix E. Then, for any $\mathbf{S}=(S, W) \in \mathcal{S}^{\mathcal{W}}(\tau)$ and $k \geq 0$,

$$
\bar{V}_{k}(\mathbf{S}, Z \equiv 0, \mathcal{M}(\tau))=0 .
$$

That is, $\mathcal{M}(\tau)$ is conditionally 0 -neutral at any $\mathbf{S}$ and for any $k \geq 0$, according to Definition 8 .

Proof. Fix $\tau$ admissible, $k \geq 0$ and define, for a given $\omega \in \Omega_{0}(\tau)$ :

$$
\begin{aligned}
\Omega_{\omega, k}(\tau)=\left\{\omega^{\prime} \in \Omega_{0}(\tau): X_{s}\left(\omega^{\prime}\right)=X_{s}(\omega) \forall s\right. & \in C \cap\left[0, \tau_{k}(\omega)\right], \\
& \text { and } \left.\tau_{i}\left(\omega^{\prime}\right)=\tau_{i}(\omega), \text { for } 0 \leq i \leq k\right\} .
\end{aligned}
$$

Lemma 5 in Appendix E, shows that $\Omega_{\omega, k}(\tau) \in \mathcal{F}_{\tau_{k}}$.

Notice that $\omega^{\prime} \in \Omega_{\omega, k}(\tau)$ implies $\mathbf{S}^{\prime}=\left(S^{\prime}, W^{\prime}\right) \in \mathcal{S}_{(\mathbf{S}, k)}(\tau)$, where $S \leftrightarrows X_{\tau}(\omega)$, $S^{\prime} \leftrightarrows X_{\tau}\left(\omega^{\prime}\right)$, this claim is obvious as we have $X_{\tau_{i}^{\prime}\left(\omega^{\prime}\right)}\left(\omega^{\prime}\right)=X_{\tau_{i}(\omega)}(\omega), 0 \leq i \leq k$. Furthermore, $X_{s \wedge \tau_{i}^{\prime}\left(\omega^{\prime}\right)}\left(\omega^{\prime}\right)=X_{s \wedge \tau_{i}(\omega)}(\omega) \forall s \in C \cap\left[0, \tau_{k}(\omega)\right]$ and so $W_{i}^{\prime}=W_{i}$, $0 \leq i \leq k$.

Given $H_{i}(S, W)=\mathbf{1}_{\Omega_{i}(\tau)}(\omega) F_{i}\left(x_{\omega, \tau_{i}}\right)$ where $F_{i}: \mathbb{R}^{C} \rightarrow \mathbb{R}$ is bounded and Borel and $S \leftrightarrows X_{\tau}(\omega)$, define $U_{i}(\omega)=\mathbf{1}_{\Omega_{i}(\tau)}(\omega) F_{i}\left(x_{\omega, \tau_{i}}\right)$ for $\omega \in \Omega_{0}(\tau)$. From the above claim, the following holds everywhere on $\Omega_{\omega, k}(\tau)$ :

$$
\begin{aligned}
{\left[-\sum_{i=k}^{M_{\tau}(\omega)-1} U_{i}(\omega)\left(X_{\tau_{i+1}}-X_{\tau_{i}}\right)(\omega)\right] } & =\left[-\sum_{i=k}^{N_{H}(\mathbf{S})-1} H_{i}(\mathbf{S})\left(S_{i+1}-S_{i}\right)\right] \\
& \leq \sup _{\tilde{\mathbf{S}} \in \mathcal{S}_{(\mathbf{S}, k)}(\tau)}\left[-\sum_{i=k}^{N_{H}(\tilde{\mathbf{S}})-1} H_{i}(\tilde{\mathbf{S}}) \Delta_{i} \tilde{\mathbf{S}}\right]
\end{aligned}
$$


Under the assumptions of Lemma 4 (b),

$$
\begin{aligned}
0 & \left.=\mathbf{1}_{\Omega_{\omega, k}(\tau)} \mathbb{E}\left[-\sum_{i=k}^{M_{\tau}-1} U_{i}\left(X_{\tau_{i+1}}-X_{\tau_{i}}\right) \mid \mathcal{F}_{\tau_{k}}\right]\right\} \\
& \left.=\mathbb{E}\left[\mathbf{1}_{\Omega_{\omega, k}(\tau)}-\sum_{i=k}^{M_{\tau}-1} U_{i}\left(X_{\tau_{i+1}}-X_{\tau_{i}}\right) \mid \mathcal{F}_{\tau_{k}}\right]\right\} \\
& \leq \mathbf{1}_{\Omega_{\omega, k}(\tau)} \sup _{\tilde{\mathbf{S}} \in \mathcal{S}_{(\mathbf{S}, k)}(\tau)}\left[-\sum_{i=k}^{N_{H}(\tilde{\mathbf{S}})-1} H_{i}(\tilde{\mathbf{S}}) \Delta_{i} \tilde{\mathbf{S}}\right] .
\end{aligned}
$$

Therefore

$$
0 \leq \sup _{\tilde{\mathbf{S}} \in \mathcal{S}_{(\mathbf{S}, k)}(\tau)}\left[-\sum_{i=k}^{N_{H}(\tilde{\mathbf{S}})-1} H_{i}(\tilde{\mathbf{S}}) \Delta_{i} \tilde{\mathbf{S}}\right]
$$

holds for all $\mathbf{S}=(S, W) \in \mathcal{S}^{\mathcal{W}}(\tau)$ and so:

$$
0 \leq \inf _{H \in \mathcal{H}} \sup _{\tilde{\mathbf{S}} \in \mathcal{S}_{(\mathbf{s}, k)}(\tau)}\left[-\sum_{i=k}^{N_{H}(\tilde{\mathbf{S}})-1} H_{i}(\tilde{\mathbf{S}}) \Delta_{i} \tilde{\mathbf{S}}\right] .
$$

As the portfolio $H_{i}=0, \forall i \geq 0$, is in $\mathcal{H}$, it follows from 6.9 that 6.8 holds.

Theorem 3 extends trivially to martingale trajectory sets of the form $\mathcal{S}^{\mathcal{W}}=$ $\cup_{\tau} \mathcal{S}^{\mathcal{W}}(\tau)$.

A function $Y: \Omega \rightarrow \mathbb{R}$ is called $\tau$-attainable if there exist an admissible $\tau$ and $U^{y} \in \mathcal{U}(\tau)$ such that

$$
Y=u_{0}^{y}+\sum_{i=0}^{M_{\tau}-1} U_{i}^{y}(\tau)\left(X_{\tau_{i+1}}-X_{\tau_{i}}\right), \quad \text { a.e. for a constant } u_{0}^{y} .
$$

Theorem 4. Consider a martingale trajectory market $\mathcal{M}(\tau)$ which satisfies the conditions of Lemma 4 in Appendix E. Let $Y$ be $\tau$-attainable and define $Z(\mathbf{S})=$ $Z_{\tau}(\mathbf{S})=Y(\omega)$ where $S \leftrightarrows X_{\tau}(\omega)$ and $\omega \in \Omega_{0}(\tau)$. Then

$$
Y_{k}(\omega)=\bar{V}_{k}(\mathbf{S}, Z, \mathcal{M}(\tau))=\underline{V}_{k}(\mathbf{S}, Z, \mathcal{M}(\tau)), \quad \forall \omega \in \Omega_{0}(\tau) \text { and } \forall 0 \leq k \leq M_{\tau(\omega)} \text {, }
$$

where $Y_{k} \equiv u_{0}^{y}+\sum_{i=0}^{k-1} U_{i}^{y}\left(X_{\tau_{i+1}}-X_{\tau_{i}}\right)$, holds everywhere on $\Omega_{0}(\tau)$. Moreover, $Y_{k}=\mathbb{E}\left(Y \mid \mathcal{F}_{\tau_{k}}\right)$ a.e. on $\Omega$ and $\mathbb{E}(\cdot)=\mathbb{E}_{Q}(\cdot)$ and $Q$ is any martingale measure equivalent to $P$.

Proof. Notice that the following holds for all $\omega \in \Omega_{0}(\tau)$ :

$$
\left.\sum_{i=0}^{k-1} U_{i}^{y}(\omega)\left(X_{\tau_{i+1}}(\omega)-X_{\tau_{i}}\right)(\omega)\right)+u_{0}^{y}=Y(\omega)-\sum_{i=k}^{M_{\tau}(\omega)-1} U_{i}^{y}(\omega)\left(X_{\tau_{i+1}}(\omega)-X_{\tau_{i}}(\omega)\right),
$$


which, given that $S \leftrightarrows X_{\tau}(\omega)$, is the same as:

$$
\sum_{i=0}^{k-1} H_{i}^{z}(\mathbf{S}) \Delta_{i} S+u_{0}^{y}=Z(\mathbf{S})-\sum_{i=k}^{N_{H}^{z}(\mathbf{S})-1} H_{i}^{z}(\mathbf{S}) \Delta_{i} S .
$$

We have used the notation $H_{i}^{z}(\mathbf{S}) \equiv \mathbf{1}_{\Omega_{i}(\tau)} F_{i}^{U^{y}}\left(x_{\omega, \tau_{i}}\right)=U_{i}^{y}(\omega)$.

Taking the conditional expectation of both sides of 6.10 with respect to $\mathcal{F}_{\tau_{k}}$ and using Lemma 4 gives

$\left.Y_{k}=\sum_{i=0}^{k-1} U_{i}^{y}\left(X_{\tau_{i+1}}-X_{\tau_{i}}\right)+u_{0}^{y}=\mathbb{E}\left(Y-\sum_{i=k}^{M_{\tau}-1} U_{i}^{y}\left(X_{\tau_{i+1}}-X_{\tau_{i}}\right)\right) \mid \mathcal{F}_{\tau_{k}}\right)=\mathbb{E}\left(Y \mid \mathcal{F}_{\tau_{k}}\right)$.

The right hand side of 6.11 is only defined a.e. on $\Omega$; in the case that is not defined everywhere on $\Omega_{0}(\tau)$ (which, we recall, is a set of probability one) we do extend $\mathbb{E}\left(Y \mid \mathcal{F}_{\tau_{k}}\right)$ to all of $\Omega_{0}(\tau)$ by means of the left hand side of (6.11).

At this point we recall Corollary 5, which is applicable to $\mathcal{M}(\tau)$ because of Theorem 3, which gives:

$$
\bar{V}_{k}(\mathbf{S}, Z, \mathcal{M}(\tau))=\underline{V}_{k}(\mathbf{S}, Z, \mathcal{M}(\tau))=\sum_{i=0}^{k-1} H_{i}^{z}(\mathbf{S}) \Delta_{i} S+u_{0}^{y}=Y_{k}(\omega),
$$

valid for all $\omega \in \Omega_{0}(\tau)$ and $\mathbf{S}=X_{\tau}(\omega)$.

Model uncertainty is usually treated by considering a subset of the set of equivalent measures. There are examples of stochastic market models for which the bounds $\inf _{Q}(Y)$ and $\sup _{Q}(Y)$ provide too large an interval in order to be informative for practical purposes. From a trajectory point of view such a situation suggests: a) a deficiency of the market model (in particular the trajectory set $\mathcal{S}$ may be too large), or b) the need to replace the super-hedging philosophy (and hence risk-free approach) for a risk taking philosophy. In this last case, the error functional used to define the bound $\bar{V}$ has to be replaced by an appropriate, trajectory based, risk-functional. There are several other logical possibilities besides a) and b), for example including liquid derivatives in the portfolio approximations (see [1]).

We have considered the set $\mathcal{P}$; it is also natural to seek an extension of the above results to the case on non-equivalent martingale measures.

\section{Conclusions And EXtensions}

The paper develops basic results on arbitrage and pricing in a general trajectory based market model. The setting naturally allows one to resort to a worst case point of view which, in turn, permits arbitrage opportunities while at the same time providing coherent prices. This fact reveals a basic extension to the classical martingale market structure. The proposed framework has also a clear conceptual and formal relationship to the well established risk-neutral approach. Given the basic nature of the arguments it is expected that extensions to other settings are possible as well. 
We have concentrated on bounding the price of an option through superhedging and underhedging, selecting an actual price inside of this interval may require to adopt a functional to accommodate the ensuing risk-taking.

Arguably, attempting a direct evaluation of the minmax optimization required in (3.1) and in related results, is a daunting task. Moreover, the minmax formulation of the problem gives no clues on how to construct the hedging values $H_{i}(S)$, for a given payoff $Z$, by means of the unfolding path values $S_{0}, S_{1}, S_{2}, \ldots$ In the paper [13], and following [5], we propose another pair of numbers, obtained through a dynamic, or iterative, definition, each instance involving a local minmax optimization. Using the new dynamic minmax definitions, [13] provides conditions under which the global and the iterated definitions coincide.

\section{Appendix A. Example}

To motivate and illustrate markets $\mathcal{M}=\mathcal{S} \times \mathcal{H}$, we introduce a family of examples modeling discretizations of stock charts. For the time being such charts are assumed to be given as a family of continuous-time functions $\mathcal{X}\left(x_{0}\right) \subseteq \mathcal{X}_{\infty}\left(x_{0}\right) \equiv$ $\left\{x \in \mathbb{R}^{[0, T]}: x(0)=x_{0}\right\}$, with $x_{0}, T>0$ and $s_{0}=e^{x_{0}}$. We will rely on some definitions.

Refining Sequence of Partitions: Consider a sequence $\left\{\Pi_{n}\right\}_{n \geq 1}$, where $\Pi_{n}=\left\{r_{i}^{n}\right\}$ is a finite partition of $[0, T]$ with $r_{0}^{n}=0$ and $\Pi_{n} \subseteq \Pi_{n+1}$. Let $\Pi \equiv \cup_{n \geq 1} \Pi_{n}$.

Selected times: Let $\mathbf{t}=\left\{t_{i}\right\}_{i \geq 0}$ be a sequence of functions $t_{i}: \mathcal{X}\left(x_{0}\right) \rightarrow \Pi$ such that $t_{0}=0$

$\forall x \in \mathcal{X}\left(x_{0}\right) \exists m(x) \in \mathbb{N}: t_{i}(x)<t_{i+1}(x)$, if $0 \leq i<m(x), t_{i}(x)=T$, if $i \geq m(x)$, and

$\forall i \geq 1$, if $\tilde{x}, x \in \mathcal{X}\left(x_{0}\right)$ with $\tilde{x}(s)=x(s)$ for $0 \leq s \leq t_{i}(x)$ then $t_{i}(\tilde{x})=t_{i}(x)$.

Observe that for any $x \in \mathcal{X}\left(x_{0}\right)$ there exists $n \geq 1$ such that $\left\{t_{i}(x)\right\}_{i \geq 0} \subset \Pi_{n}$. This is so because $t_{i}(x) \in \Pi$ implies that there exists $n_{i}$, the minimum such that $t_{i}(x) \in \Pi_{n_{i}}$, and $n=\max \left\{n_{i}: 0 \leq i \leq m(x)\right\}$.

Let $\mathcal{R}=(\Pi, \mathbf{t})$, and define the following general class of discrete trajectories:

$$
\mathcal{S}\left(s_{0}, \mathcal{X}, \mathcal{R}\right) \equiv\left\{S=\left\{S_{i}\right\}_{i \geq 0}: S_{i}=\exp \left(x\left(t_{i}\right)\right), i \geq 0, \text { for some } x \in \mathcal{X}\left(x_{0}\right)\right\},
$$

$\left(t_{i}=t_{i}(x)\right)$.

General aspects. A refining sequence of partitions reflects a financial situation where the investor re-balances her/his portfolio with a certain minimum time resolution but is willing to refine it further if deemed necessary. The case of a fixed partition $\left(\Pi_{n}\right.$ the same for all $\left.n\right)$ means that the investor will never rebalance more often than an a-priori given time resolution.

There is no essential result in our paper that requires $S_{i} \geq 0$, so there is no need to use the exponential function in the definition $S_{i}=e^{x\left(t_{i}\right)}$ but, doing so makes it easier to connect with the usual geometric stochastic models as well as with [5].

We are interested in prescribing "structured" subsets of $\mathcal{S}\left(s_{0}, \mathcal{X}, \mathcal{R}\right)$, we do this by means of an observable functional $F$. For simplicity, the functional could be 
defined on $\mathcal{X}\left(x_{0}\right)$ (and could depend on other variables as well) and takes values on $\mathbb{R} \cup\{\infty\}$. As a particular case, the functional $F$ selects those $x \in \mathcal{X}\left(x_{0}\right)$ such that $x$ has finite quadratic variation in the interval $[s, t]$ respect to $\Pi$, that is, the following limit exists

$$
F(x, s, t)=\lim _{n \rightarrow \infty} \sum_{s \leq r_{i}^{n}, r_{i+1}^{n} \leq t}\left(x\left(r_{i+1}^{n}\right)-x\left(r_{i}^{n}\right)\right)^{2} .
$$

Other observable, additive, non-decreasing and non-negative quantities could be used as well, for example, the number of transactions from $t_{0}=0$ to $t$, or the total number of shares transacted from $t_{0}=0$ to $t$. Intuitively, each time a transaction takes place, the value for the observable quantity represents the tick of a trajectory based clock (usually interpreted as a "business clock" with trajectory dependent rate.)

The following is an example of a structured discrete trajectory set. For $c, d>0$ real numbers and $Q \subset(0, \infty)$, define

$$
\begin{aligned}
\mathcal{S}\left(s_{0}, c, d, Q\right)=\{S & \in \mathcal{S}\left(s_{0}, \mathcal{X}, \mathcal{R}\right):\left|x\left(t_{i+1}\right)-x\left(t_{i}\right)\right| \leq d, \\
& \left.F\left(x, t_{i}, t_{i+1}\right) \leq c, F\left(x, t_{0}, t_{m(x)}\right) \in Q, 0 \leq i<m(x)\right\} .
\end{aligned}
$$

For the case of the functional given by A.1), the requirements defining $\mathcal{S}\left(s_{0}, c, d, Q\right)$ can be interpreted as imposing constraints on the maximum consumed quadratic variation and on the maximum absolute value of the change on chart values, both in between consecutive trading instances. In addition, the condition $F\left(x, t_{0}, t_{m(x)}\right) \in$ $Q$ means that we deal with trajectories whose total quadratic variation in the interval $[0, T]$ belongs to the a-priori given subset $Q$. In effect, the imposed constraints restrict the outcomes resulting from the interaction between market fluctuations and portfolio rebalances.

A.1. Construction of trajectory sets from augmented data. Here we describe a set of trajectories that does not require a continuous time model for the charts, we rely on the formalism from Section 2.2 The general principle guiding the construction is to isolate an observable quantity (representing a variable of interest) and proceed to define a trajectory space by imposing constraints relating the trajectories and a free variable representing this observable. We work with observables given by a functional, still denoted by $F$, but now defined on charts (i.e., the values of some unfolding financial data), $F$ may also depend on other variables as well.

The definition of $\mathcal{S}\left(s_{0}, c, d, Q\right)$ in $\mathrm{A} .2$ depends on having access to the functions $x \in \mathcal{X}$. We now turn the tables around and re-define $\mathcal{S}\left(s_{0}, c, d, Q\right)$ as $\mathcal{S}^{\mathcal{W}}\left(s_{0}, c, d, Q\right)$, a set which does not require any reference to a given class of continuous-time trajectories. We still allow observable charts to unfold in continuoustime. Trajectories are given by a sequence of tuples $\mathbf{S}=\left\{\left(S_{i}, W_{i}\right)\right\}_{i \geq 0}$, which will be associated to samples of continuous-time charts. This is a natural way to proceed; information deemed important for modelling is lost when sampling, hence this information will be encoded by the variable $W_{i}$ (associated to the functional $F$ ). 
The definition below assumes given: $w_{0}=0, s_{0}$ and $c, d>0$ real numbers, $\sum_{i} \subseteq \mathbb{R}$ and sets $Q, \Omega_{i} \subset(0, \infty)$.

Definition 14. $\mathcal{S}^{\mathcal{W}}\left(s_{0}, d, c, Q\right)$ will denote a subset of $\mathcal{S}_{\infty}^{\mathcal{W}}\left(s_{0}, w_{0}\right)$ so that $\mathbf{S} \in$ $\mathcal{S}^{\mathcal{W}}\left(s_{0}, d, c, Q\right)$ satisfies $S_{i} \in \Sigma_{i}, W_{i} \in \Omega_{i}$ and:

(1) $\left|\log S_{i+1}-\log S_{i}\right| \leq d$ for all $i \geq 0$,

(2) $0<W_{i+1}-W_{i} \leq c$ for all $i \geq 0$.

Moreover, there exists at least one $i^{*}$ such that

(3) $W_{i^{*}} \in Q$.

Associated markets $\mathcal{S}^{\mathcal{W}}\left(s_{0}, c, d, Q\right) \times \mathcal{H}$ are required to satisfy: If $H \in \mathcal{H}$ then $W_{N_{H}(\mathbf{S})} \in Q$.

Remark 6. As already mentioned, the condition $\left|\log S_{i+1}-\log S_{i}\right| \leq d$ could equally be replaced by $\left|S_{i+1}-S_{i}\right| \leq d$ (of course with an appropriately chosen value for $d$ ).

We emphasize that $\mathcal{S}^{\mathcal{W}}\left(s_{0}, d, c, Q\right)$, as characterized above, does not need to be, in general, the set of all trajectories $\mathbf{S}$ satisfying the listed constraints in Definition (14); specific examples are described in [13. Comparing with A.2), we see that we have allowed $F$ to be an independent variable $W$. For $\mathbf{S} \in \mathcal{S}^{\mathcal{W}}\left(s_{0}, d, c, Q\right)$ there could be multiple indexes $i^{*}$.

The set $\mathcal{S}^{\mathcal{W}}\left(s_{0}, c, d, Q\right)$ is used for modelling the unfolding of a data chart $x\left(t_{i}\right)$ by mapping $\left\{\left(e^{x\left(t_{i}\right)}, F\left(x, t_{0}, t_{i}\right)\right)\right\}$, one index $i$ at a time (i.e., as the chart unfolds), to its closest path $\left\{\left(S_{i}, W_{i}\right)\right\}_{i \geq 0}$.

The trajectory set introduced in [5] can be recovered as a special case of Definition 14 by taking $Q=\left\{v_{0}\right\}$ and defining

$$
W_{i}=\sum_{k=0}^{i-1}\left(\log S_{k+1}-\log S_{k}\right)^{2} ;
$$

moreover, we need to require the existence of $i^{*}$ satisfying $W_{i^{*}}=v_{0}$. Therefore $W_{i+1}-W_{i}=\left(\log S_{i+1}-\log S_{i}\right)^{2}$ and the constraint $0<W_{i+1}-W_{i} \leq c$ in Definition 14 corresponds to $c=d^{2}$. Moreover, as $W_{i}$ depends on $S_{k}, 0 \leq k \leq i$, there is no need to work with tuples $\left(S_{i}, W_{i}\right)$ in this case. Not imposing A.3 allows to incorporate 0-neutral nodes which are arbitrage nodes (see Definition 10 and related comments afterwards.) An analysis of these considerations in the context of the example is outside the scope of the paper, details are given in 13 .

A natural discretization leading to an implementation of $\mathcal{S}^{\mathcal{W}}\left(s_{0}, c, d, Q\right)$ is obtained by introducing real numbers $\delta, \beta>0$. The coordinates $S_{i}$ are then restricted to belong to the sets $\Sigma_{i}=\Sigma(\delta) \equiv\left\{s_{0} e^{k \delta}, k \in \mathbb{Z}\right\}$ and $W_{i}$ to $\Omega_{i} \equiv \Omega(\beta)=\left\{j \beta^{2}, j \in\right.$ $\mathbb{N}\}$, thus $Q$ is now a set $Q(\beta) \subseteq \Omega(\beta)$.

Local behavior. The way of defining trajectory sets $\mathcal{S}^{\mathcal{W}}\left(s_{0}, d, c, Q\right)$, or their finite versions, will make it easy to check if the local properties of 0-neutral or up-down are satisfied. This is so because our constraints are given locally (i.e., at each node) and the combinatorial definitions will allow trajectories to move up or down. Next, 
as an illustration, we provide a general argument on how to argue for the validity of these local properties.

Assume that the the sets $\Sigma_{i}$ in the trajectory space $\mathcal{S}^{\mathcal{W}}\left(s_{0}, c, d, Q\right)$ of Definition 14 do not attain minimum nor maximum and fix a node $\left(S_{i}, W_{i}\right)$ of a trajectory $\mathbf{S}$. Clearly, there exists the possibility of choosing trajectories $\tilde{\mathbf{S}}, \hat{\mathbf{S}} \in \mathcal{S}_{(\mathbf{S}, i)}^{\mathcal{W}}$ such that $\tilde{S}_{i+1}>S_{i}$, and $\hat{S}_{i+1}<S_{i}$ respectively, so any node is up-down, and in that case the market results locally arbitrage-free, see Definition 10 Specific instances of the sets $\mathcal{S}^{\mathcal{W}}\left(s_{0}, c, d, Q\right)$ or their discrete or finite versions will impose further constraints beyond the ones listed in Definition 14. In each such case, we will need to check the validity of the needed local requirements, 0-neutrality or arbitrage-free, so that our results hold.

For display purposes, consider a finite space $\mathcal{S}^{\mathcal{W}}\left(s_{0}, c, d, Q\right)$ consisting of all trajectories satisfying the conditions in Definition 14 with $N_{2}=N_{1}=100, \beta=$ $\delta=0.0082, Q=\left\{N_{2} \beta^{2}\right\}, c=d^{2}, s_{0}=1, p=3$ (and so $d=0.0246$ ). Figure 1 shows 200 random samples of trajectories from such trajectory space. Figure 2 shows random samples of trajectories from two conditional spaces from the above trajectory space.

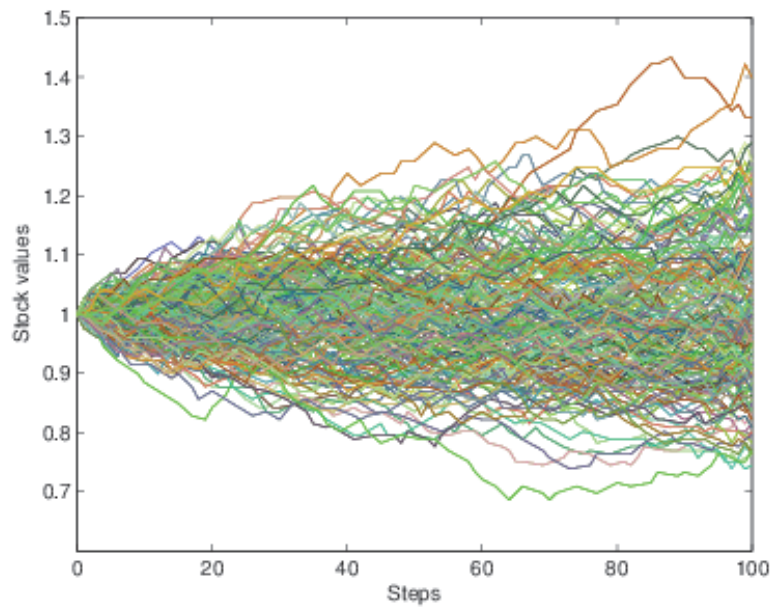

FiguRE 1. Samples from trajectory space.

\section{Appendix B. Connection with robust literature}

This appendix relies on notation and results from [6] and [7]. Reference [7] proves a dual representation for the superhedging functional $\bar{V}(\cdot)$; the results are only presently available for finitely many integer times $t=0, \ldots, T$.

We rely on notation from the mentioned references; we have replaced their stochastic process notation $\left\{S_{t}\right\}$ by $\left\{X_{t}\right\}$, to avoid confusion with our trajectory notation $S$. For the sake of brevity, we refer to these references for some of the 


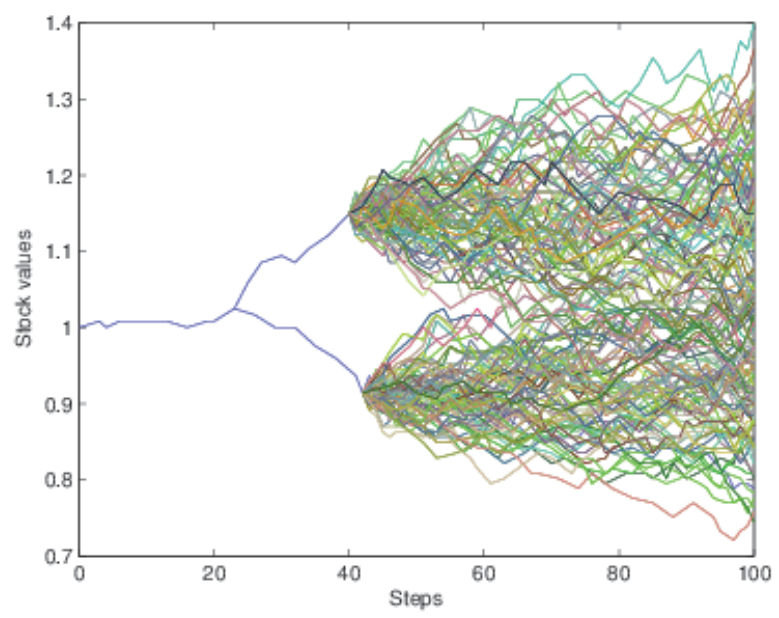

FiguRE 2. Sampled trajectories from conditional spaces.

notions that we have to rely upon. Also, the notation $\mathcal{M}$ from [6] and [7] has a specific meaning (as described below) for this appendix only (i.e., unrelated to its use elsewhere in the paper).

Let $\Omega$ be a Polish space with $\mathcal{F} \equiv \mathcal{B}(\Omega)$ the Borel sigma algebra. Let $X=\left\{X_{t}\right\}$ be random variables on $\Omega$; let $\mathbb{F}^{X}=\left\{\mathcal{F}_{t}^{X}\right\}$ be the canonical filtration and consider the universal filtration $\mathbb{F}=\left\{\mathcal{F}_{t}\right\}$, containing $\mathbb{F}^{X}([7)$.

Let $\mathcal{N}$ (here, again, we depart from the notation used in [7]) be the set of probability measures $Q$ on the measure space $(\Omega, \mathcal{F})$ such that $X$ is a $(Q, \mathbb{F})$ martingale Let $\mathcal{N}_{+} \subseteq \mathcal{N}$ be such that if $Q \in \mathcal{N}_{+}$then $\operatorname{supp}(Q)=\Omega($ where $\operatorname{supp}(Q)$ denotes the (topological) support of the measure $Q$ ).

With some abuse of notation let $\mathcal{H}$ denote the class of $\mathbb{F}$-predictable processes $H=\left\{H_{t}\right\}$. For the next result it is necessary to introduce another definition of local arbitrage. That concept represents the situation when we know a trajectory and an instance where an arbitrage opportunity will arise. It also assumes the existence of a portfolio that takes advantage of the arbitrage opportunity. if

$A$ discrete market model $\mathcal{M}=\mathcal{S} \times \mathcal{H}$ is said to have a node of arbitrage $(S, j, H)$

$$
\inf _{\tilde{S} \in \mathcal{S}_{(S, j)}}\left[H_{j}(S) \Delta_{j} \tilde{S}\right] \geq 0 \quad \text { and } \quad \sup _{\tilde{S} \in \mathcal{S}_{(S, j)}}\left[H_{j}(S) \Delta_{j} \tilde{S}\right]>0
$$

The logical negation of the conditions in (B.1) will give local sufficient conditions leading to (global) no arbitrage results: 
$A$ discrete market $\mathcal{M}$ is said free of nodes of arbitrage if it has no nodes of arbitrage at any $(S, H, j)$, that is, the following holds at any node $(S, H, j)$ :

$$
\inf _{\tilde{S} \in \mathcal{S}_{(S, j)}}\left[H_{j}(S) \Delta_{j} \tilde{S}\right]<0
$$

or

$$
\sup _{\tilde{S} \in \mathcal{S}_{(S, j)}}\left[H_{j}(S) \Delta_{j} \tilde{S}\right] \leq 0
$$

Remark 7. Notice that above we refer to $\mathcal{M}$ as being free of nodes of arbitrage, this is in contrast to saying, in Definition 10 that $\mathcal{S}$ is locally arbitrage-free. The obvious relationship is spelled out in Proposition 9 below.

Proposition 9. Consider a trajectory space $\mathcal{S}$ that is locally arbitrage-free (as per Definition 10) and $\mathcal{H}$ a portfolio set. Then, $\mathcal{M}=\mathcal{S} \times \mathcal{H}$ is free of nodes of arbitrage (as per equations (B.2) and (B.3).

Proof. Fix $H \in \mathcal{H}, S \in \mathcal{S}$ and $j \geq 0$. If $H_{j}(S)=0$, B.3 is clearly verified. While if $H_{j}(S) \neq 0$, then $(\mathrm{B} .2)$ or $(\mathrm{B} .3)$ are satisfied, whenever either 4.2 or $(4.3)$ are valid.

The following proposition connects our setting with results from [6] and [7].

Proposition 10. Let $\mathcal{S}$ be a trajectory set, and assume $\Omega \equiv \mathcal{S}$ to be Polish and $\mathcal{H}$ as defined above. Set $N_{H}(\omega)=T$, for all $\omega \in \Omega$ and $H \in \mathcal{H}$. Then, if $\Omega$ is locally arbitrage-free and $Z$ is $\mathcal{F}$-measurable:

$$
\bar{V}(Z)=\sup _{Q \in \mathcal{N}} \mathbb{E}_{Q}(Z)
$$

and $\mathcal{N}_{+} \neq \emptyset$.

Proof. To obtain (B.4) we will apply Theorem 1.1 from [7] to the process $X=\Pi=$ $\left\{\Pi_{t}\right\}$, with $\Pi_{t}(S)=S_{t}$. From this theorem and the definition of $\bar{V}(Z)$, equation (B.4) will follow if $\Omega_{*}=\Omega\left(\Omega_{*}\right.$ as defined in [7]). Our assumption on $\Omega$ being locally arbitrage-free implies, by Proposition 9 that $\mathcal{H}$ has no 1 -point arbitrage (as in [7]). Therefore, from Proposition 4.1 in [6] and its proof, it follows that the no 1-point arbitrage property implies $\Omega_{*}=\Omega$ and $\mathcal{N}_{+} \neq \emptyset$ as well.

Apparently the above results also hold if one starts initially with a larger filtration $\left\{\mathcal{G}_{t}\right\}$ containing the canonical filtration and constructs the associated universal filtration associated to such initial filtration. If that case, the above proposition extends to the situation when $\Omega \equiv \mathcal{S}^{\mathcal{W}}$ and if $\left\{\mathcal{G}_{t}\right\}$ is a filtration containing the canonical filtration $\mathcal{G}^{S}=\left\{\mathcal{G}_{t}^{S}\right\}$, the latter generated by the process $\Pi=\left\{\Pi_{t}\right\}$, where $\Pi_{t}(\mathbf{S}) \equiv S_{t}, t=0,1, \ldots$, with $\mathbf{S}=\left\{\left(S_{i}, W_{i}\right)\right\}_{i \geq 0}$. So each $\Pi_{t}: \Omega \rightarrow \mathbb{R}$ is $\mathcal{G}_{t}$-measurable.

Also observe that in [6, Lemma 3.6] it is proven that $H \in \mathcal{H}$ is a 1 p-arbitrage if and only if $(S, H, t)$ is a node of arbitrage, for some $S \in \mathcal{S}$ and $t \in\{1, \ldots, T\}$. Consequently, if $\mathcal{M}$ is free of nodes of arbitrage the conclusion of Proposition 10 follows. 
The existence of 1-point arbitrage is the weakest possible arbitrage hence the absence of such arbitrage is the strongest notion of no arbitrage considered in [6]. It guarantees the existence of a martingale measure having as support the original trajectory space.

\section{Appendix C. Other CONDITIONS to have A PRICE INTERVAL}

The following definition reflects the situation of an investor who decides conditionally on a bounded number of transactions, that he/she will stop trading after a certain fixed number of future trades. The setting allows for unbounded $N_{H}$.

Definition 15 (Initially Bounded). Given a market $\mathcal{M}=\mathcal{S} \times \mathcal{H}$ and $H \in \mathcal{H}$; we will call $N_{H}$ initially bounded if there exists a bounded function $\rho: \mathcal{S} \rightarrow \mathbb{N}$ (which may depend on $H$ ) such that for all $S \in \mathcal{S}$ :

$$
N_{H} \text { is bounded on } \mathcal{S}_{(S, \rho(S))} \text {. }
$$

If $N_{H}$ is bounded, then it is actually initially bounded by taking $\rho=N_{H}$.

The above definition gives a setting ensuring that a market $\mathcal{M}$ is 0 -neutral. For reasons of space, we omit the proof of the following theorem, a particular case of which was established in Corollary 1 . Section 4.

Theorem 5. Given a market $\mathcal{M}=\mathcal{S} \times \mathcal{H}, k \geq 0$ and $S^{k} \in \mathcal{S}$, assume that $N_{H}$ is initially bounded for any $H \in \mathcal{H}$ and each node $(S, j)$, with $S \in \mathcal{S}_{\left(S^{k}, k\right)}$ and $j \geq k$, is 0 -neutral. Then $\mathcal{M}$ is conditionally 0 -neutral at $\left(S^{k}, k\right)$.

The following corollary provides existence of the pricing interval in the setting of Theorem 5. The sum of portfolios, used in the proof, is presented preceding Lemma 2 in Appendix D

Corollary 8. Consider a discrete market $\mathcal{M}=\mathcal{S} \times \mathcal{H}$, a function $Z$ defined on $\mathcal{S}, S \in \mathcal{S}$ and $k \geq 0$ fixed. Assume $N_{H}$ to be initially bounded for all $H \in \mathcal{H}$ and either $N_{H}$ is a stopping time for all $H \in \mathcal{H}$ or all $H \in \mathcal{H}$ are liquidated. Then, if each node $(\tilde{S}, j)$, with $\tilde{S} \in \mathcal{S}_{(S, k)}$ and $j \geq k$, is 0 -neutral:

$$
\underline{V}_{k}(S, Z, \mathcal{M}) \leq \bar{V}_{k}(S, Z, \mathcal{M}) \text {. }
$$

Proof. Observe that the initially bounded property is closed under addition. Indeed, let $\rho_{1}, \rho_{2}$ be the functions required by Definition 15 for $H^{1}, H^{2} \in \mathcal{H}$ respectively. Then, set $H=H^{1}+H^{2}$ and $\rho \equiv \max \left\{\rho_{1}, \rho_{2}\right\} ;$ since $\mathcal{S}_{(S, \rho(S))} \subset \mathcal{S}_{\left(S, \rho_{j}(S)\right)}$, $j=1,2$, and so $N_{H}$ is bounded in $\mathcal{S}_{(S, \rho(S))}$. Therefore, Theorem 5 applies implying $\widetilde{\mathcal{M}}=\mathcal{S} \times(\mathcal{H}+\mathcal{H})$ is conditionally 0 -neutral at $(S, k)$. It follows that

$$
\underline{V}_{k}(S, Z, \mathcal{M}) \leq \underline{V}_{k}(S, Z, \widetilde{\mathcal{M}}) \leq \bar{V}_{k}(S, Z, \widetilde{\mathcal{M}}) \leq \bar{V}_{k}(S, Z, \mathcal{M}),
$$

where the innermost inequality follows from Theorem 1

Remark 8. A more basic result is concealed in the proof of the last corollary, indeed, under those hypotheses $\mathcal{S} \times(\mathcal{H}+\mathcal{H})$ is conditionally 0-neutral. 


\section{Appendix D. Further Properties of PRICE BOUNDS}

The following developments are stated and proven with two portfolio sets $\mathcal{H}^{1}, \mathcal{H}^{2}$, the reader could take $\mathcal{H}=\mathcal{H}^{1}=\mathcal{H}^{2}$, as the extra generality is not used in the rest of the paper.

Define

$$
\mathcal{H}^{1}+\mathcal{H}^{2}=\left\{H^{1}+H^{2}: H^{1} \in \mathcal{H}^{1}, H^{2} \in \mathcal{H}^{2}\right\},
$$

where the sum $H \equiv H^{1}+H^{2}$ is defined as follows:

$$
\begin{gathered}
N_{H} \equiv \max \left\{N_{H^{1}}, N_{H^{2}}\right\}, \\
H_{i}= \begin{cases}H_{i}^{1}+H_{i}^{2} & \text { if } 0 \leq i<\min \left\{N_{H^{1}}, N_{H^{2}}\right\}, \\
H_{i}^{j} & \text { if } \min \left\{N_{H^{1}}, N_{H^{2}}\right\} \leq i,\end{cases}
\end{gathered}
$$

where $N_{H}$ is attained in $H^{j}, j=1$ or $j=2$. We now check that the portfolio sum $H$ is non-anticipative under the assumption that $N_{H^{j}}, j=1,2$, are stopping times. Let $S_{j}^{\prime}=S_{j}, 0 \leq j \leq i$; if $i<\min \left\{N_{H^{1}}(S), N_{H^{2}}(S)\right\} \equiv m$, it is clear that $H_{i}(S)=H_{i}\left(S^{\prime}\right)$. Consider then $i \geq m$ and assume, without lost of generality, that $N_{H}^{1}(S)=m$, then $N_{H^{1}}\left(S^{\prime}\right)=N_{H^{1}}(S)$. If $N_{H^{2}}\left(S^{\prime}\right)<N_{H^{1}}\left(S^{\prime}\right)$, it would result that $S_{j}^{\prime}=S_{j}, 0 \leq j \leq N_{H^{2}}\left(S^{\prime}\right)$, and so $N_{H^{2}}\left(S^{\prime}\right)=N_{H^{2}}(S) \geq N_{H^{1}}(S)=N_{H^{1}}\left(S^{\prime}\right)$, a contradiction.

In case that the functions $N_{H^{j}}$ are not stopping times, the portfolio sum $H$ is still non-anticipative if liquidation is assumed. Indeed, if the portfolios $H^{j}$ are liquidated at $N_{H^{j}}, j=1,2$ (i.e., for any $S \in \mathcal{S}, H_{i}^{j}(S)=0$ for $i \geq N_{H^{j}}(S)$ ), the sum definition in (D.1) reduces to

$$
H_{i}=H_{i}^{1}+H_{i}^{2} \quad \text { for any } i \geq 0 .
$$

It is clear that if $S, S^{\prime} \in \mathcal{S}$ with $S_{j}^{\prime}=S_{j}, 0 \leq j \leq i$, for some $i \geq 0$, then $H_{i}\left(S^{\prime}\right)=H_{i}^{1}\left(S^{\prime}\right)+H_{i}^{2}\left(S^{\prime}\right)=H_{i}^{1}(S)+H_{i}^{2}(S)=H_{i}(S)$.

Observe also that for any $S \in \mathcal{S}$ and $k \geq 0$,

$$
\sum_{i=k}^{N_{H}(S)-1} H_{i}(S) \Delta_{i} S=\sum_{i=k}^{N_{H^{1}}(S)-1} H_{i}^{1}(S) \Delta_{i} S+\sum_{i=k}^{N_{H^{2}}(S)-1} H_{i}^{2}(S) \Delta_{i} S .
$$

Lemma 2. Let $\mathcal{M}^{1}=\mathcal{S} \times \mathcal{H}^{1}$ and $\mathcal{M}^{2}=\mathcal{S} \times \mathcal{H}^{2}$ be markets, and assume either: for all $H^{j} \in \mathcal{H}^{j}, j=1,2, N_{H^{j}}$ are stopping times or all $H^{j} \in \mathcal{H}^{j}, j=1,2$, are liquidated. Set $\widetilde{\mathcal{M}}=\mathcal{S} \times\left(\mathcal{H}^{1}+\mathcal{H}^{2}\right)$ and $S \in \mathcal{S}$ and $0 \leq k$. Assume $Z_{1}, Z_{2}, Z$ are real valued functions defined on $\mathcal{S}$; then

$$
\bar{V}_{k}\left(S, Z_{1}+Z_{2}, \widetilde{\mathcal{M}}\right) \leq \bar{V}_{k}\left(S, Z_{1}, \mathcal{M}^{1}\right)+\bar{V}_{k}\left(S, Z_{2}, \mathcal{M}^{2}\right) .
$$

Moreover, if $\widetilde{\mathcal{M}}$ is conditional 0-neutral at $(S, k)$ then

$$
\underline{V}_{k}\left(S, Z, \mathcal{M}^{1}\right) \leq \bar{V}_{k}\left(S, Z, \mathcal{M}^{2}\right) .
$$


Proof. Let $H^{j}$ be generic elements of $\mathcal{H}^{j}, j=1,2$, so $H^{1}+H^{2} \in \mathcal{H}^{1}+\mathcal{H}^{2}$. Then

$$
\begin{aligned}
& \bar{V}_{k}\left(S, Z_{1}+Z_{2}, \widetilde{\mathcal{M}}\right) \\
& \leq \sup _{\tilde{S} \in \mathcal{S}_{(S, k)}}\left[Z_{1}(\tilde{S})-\sum_{i=k}^{N_{H^{1}}(\tilde{S})-1} H_{i}^{1}(\tilde{S}) \Delta_{i} \tilde{S}+Z_{2}(\tilde{S})-\sum_{i=k}^{N_{H^{2}}(\tilde{S})-1} H_{i}^{2}(\tilde{S}) \Delta_{i} \tilde{S}\right] \\
& \leq \sup _{\tilde{S} \in \mathcal{S}_{(S, k)}}\left[Z_{1}(\tilde{S})-\sum_{i=k}^{N_{H^{1}}(\tilde{S})-1} H_{i}^{1}(\tilde{S}) \Delta_{i} \tilde{S}\right]+\sup _{\tilde{S} \in \mathcal{S}_{(S, k)}\left[Z_{2}(\tilde{S})-\sum_{i=k}^{N_{H^{2}}(\tilde{S})-1} H_{i}^{2}(\tilde{S}) \Delta_{i} \tilde{S}\right] .}
\end{aligned}
$$

Therefore, taking infimum over $\mathcal{H}^{1}$ and $\mathcal{H}^{2}$,

$$
\bar{V}_{k}\left(S, Z_{1}+Z_{2}, \widetilde{\mathcal{M}}\right) \leq \bar{V}_{k}\left(S, Z_{1}, \mathcal{M}^{1}\right)+\bar{V}_{k}\left(S, Z_{2}, \mathcal{M}^{2}\right) .
$$

This proves (D.2). For (D.3), by the result on $\left(\mathrm{D} .2\right.$ with $Z_{1}=-Z$ and $Z_{2}=Z$, and the conditional 0 -neutral property of $\widetilde{\mathcal{M}}$ we have

$$
0=\bar{V}_{k}(S, 0, \widetilde{\mathcal{M}}) \leq \bar{V}_{k}\left(S,-Z, \mathcal{M}^{1}\right)+\bar{V}_{k}\left(S, Z, \mathcal{M}^{2}\right),
$$

which gives the desired result.

D.1. Minmax functions. Conditions for boundedness of $\underline{V}(Z)$ and $\bar{V}(Z)$. The general setting works with a general function $Z: \mathcal{S} \rightarrow \mathbb{R}$ but, in order to link to finance and guarantee finiteness of the bounds, we introduce sufficient conditions through the (so called) minmax functions.

The function $Z$ is called a European option when there exist an integer $M$, a function $\hat{Z}: \mathbb{R}^{M} \rightarrow \mathbb{R}$, and stopping times $\tau_{i} \leq \tau_{i+1}, i=1, \ldots, M$, so that $Z(S)=\hat{Z}\left(S_{\tau_{1}(S)}, \ldots, S_{\tau_{M}(S)}\right) . \hat{Z}$ will be called a payoff; the setting allows for path dependency. For a European call or put option (and so $M=1$ ) portfolios in $\mathcal{H}$ could/should be required to satisfy $N_{H}(S) \leq \tau_{1}(S)$ for all $H \in \mathcal{H}$ and for all $S$.

Definition 16 (Upper and Lower Minmax Functions). Given a finite sequence of stopping times $\left(\nu_{i}\right)_{i=1}^{n}$ with $\nu_{i}<\nu_{i+1}$ for $1 \leq i<n$, a real sequence $\left(a_{i}\right)_{i=1}^{n}$, and $b \in \mathbb{R}$, we call $Z$ an upper minmax function if

$$
Z(S) \leq \sum_{i=1}^{n} a_{i} S_{\nu_{i}(S)}+b, \quad \forall S \in \mathcal{S} .
$$

Similarly, $Z$ is called a lower minmax function if

$$
Z(S) \geq \sum_{i=1}^{n} a_{i} S_{\nu_{i}(S)}+b, \quad \forall S \in \mathcal{S} .
$$

Examples. The following examples show that some common options belong to the class of minmax functions.

(1) If $Z$ is a European call option with strike price $K>0$ and $N(S)$ a stopping time, $Z(S)=\left(S_{N(S)}-K\right)^{+} \leq S_{N(S)}$, then $Z$ is an upper minmax function with $n=1, a_{1}=1, \nu_{1}(S)=N(S)$, and $b=0$. 
(2) If $Z$ is a European put option with strike price $K>0$ and $N(S)$ a stopping time, $Z(S)=\left(K-S_{N(S)}\right)^{+} \leq K$, then $Z$ is an upper minmax function with $n=1$, $a_{1}=0$ and $b=K$.

Clearly, the above two examples are also lower minmax functions.

(3) Under the assumption $S_{k} \geq 0$ for all $S \in \mathcal{S}$ and all $k \geq 0$, if $Z(S)=$ $a \max _{1 \leq i \leq n} S_{\nu_{i}(S)}+b$ with $a>0$, then $Z(S) \leq \sum_{i=1}^{n} a S_{\nu_{i}(S)}+b$ and so $Z$ is an upper minmax function with $a_{i}=a$ for all $i=1, \ldots, n$.

(4) If $Z(S)=\frac{1}{n} \sum_{i=1}^{n} S_{\nu_{i}(S)}$, then $Z$ is an upper minmax function with $a_{i}=\frac{1}{n}$ for all $i=1, \ldots, n$ and $b=0$.

Notice that, in particular, if $S_{j}$ is uniformly bounded from below by a constant, for all $j$, then examples (3) and (4) are lower minmax functions as well.

Remark 9. Under some assumptions on the market $\mathcal{M}$, such as conditionally 0 neutral, it can be proven that the conditional bounds $\bar{V}_{k}(S, Z, \mathcal{M})$ and/or $\underline{V}_{k}(S, Z, \mathcal{M})$ are finite when $Z$ is an upper or lower minmax function; for reasons of space, details are provided elsewhere ([13]).

\section{Appendix E. Connections With Risk neutral pricing. Auxiliary} MATERIAL

Lemma 3. The function $\phi:\left(\Omega, \mathcal{F}_{\tau_{i}}\right) \rightarrow\left(\mathbb{R}^{C}, \mathcal{B}\left(\mathbb{R}^{C}\right)\right)$ defined by $\phi(\omega)=x_{\omega, \tau_{i}}$ is measurable.

Proof. For $1 \leq j \leq n$, fix $c_{j} \in C, \Gamma_{j} \in \mathcal{B}(\mathbb{R})$ and let $\mathcal{C}=\left\{x \in \mathbb{R}^{C}: x\left(c_{j}\right) \in \Gamma_{j}, 1 \leq\right.$ $j \leq n\}$. Thus

$$
\phi^{-1}(\mathcal{C})=\cap_{j=1}^{n}\left\{\omega: x_{\omega, \tau_{i}}\left(c_{j}\right) \in \Gamma_{j}\right\}=\cap_{j=1}^{n} X_{c_{j} \wedge \tau_{i}}^{-1}\left(\Gamma_{j}\right) .
$$

For showing that $\phi^{-1}(\mathcal{C}) \in \mathcal{F}_{\tau_{i}}$, it is then enough to prove that, for any $c \in C$ and $\Gamma \in \mathcal{B}(\mathbb{R}), X_{c \wedge \tau_{i}}^{-1}(\Gamma) \in \mathcal{F}_{\tau_{i}}$. This happens if, for any $t \geq 0$,

$$
A=\left\{\omega: X_{c \wedge \tau_{i}(\omega)}(\omega) \in \Gamma, \tau_{i}(\omega) \leq t\right\} \in \mathcal{F}_{t} .
$$

To prove this, let us first define $B=\left\{\omega: X_{\tau_{i}(\omega)}(\omega) \in \Gamma, \tau_{i}(\omega) \leq t\right\}$ and consider two cases.

I. Assume $t \leq c$, then for $\omega \in A, c \wedge \tau_{i}(\omega)=\tau_{i}(\omega)$ which implies that $A \subset B$. Conversely, if $\omega \in B$ then $c \wedge \tau_{i}(\omega)=\tau_{i}(\omega)$, and $B \subset A$. Now we are going to prove that

$$
B=\cup_{\{s \in C: s \leq t\}}\left\{\omega: \tau_{i}(\omega)=s, X_{s}(\omega) \in \Gamma\right\} .
$$

Indeed, if $\omega \in B$ there exists $s \in C$ such that $s=\tau_{i}(\omega) \leq t$ and then $X_{\tau_{i}(\omega)}(\omega)=$ $X_{s}(\omega) \in \Gamma$. The converse is also clearly true. Finally, since for each $s \in C, s \leq t$ we have $\left\{\omega: \tau_{i}(\omega)=s, X_{s}(\omega) \in \Gamma\right\} \in \mathcal{F}_{s} \subset \mathcal{F}_{t}$, it follows that $A \in \mathcal{F}_{t}$.

II. The case when $c<t$ follows from this decomposition of $A$ :

$$
A=\left(\left\{\omega: \tau_{i}(\omega) \leq c\right\} \cap B\right) \cup\left(\left\{\omega: \tau_{i}(\omega)>c\right\} \cap\left\{\omega: X_{c}(\omega) \in \Gamma, \tau_{i}(\omega) \leq t\right\}\right) .
$$

Since $\left\{\omega: \tau_{i}(\omega) \leq c\right\},\left\{\omega: \tau_{i}(\omega)>c\right\},\left\{\omega: X_{c}(\omega) \in \Gamma\right\} \in \mathcal{F}_{c} \subset \mathcal{F}_{t}$ and $B \in \mathcal{F}_{t}$, $A \in \mathcal{F}_{t}$ as well. 
Recall that $\mathcal{P}=\mathcal{P}(P)$ is the set of all martingale probability measures equivalent to $P$ and $\mathbb{E}_{Q}(Y)$ denotes expectation with respect to the probability measure $Q$.

Lemma 4. Let $Q \in \mathcal{P}(P)$ and assume, for $i \geq 0$, that $U_{i}$ are $\mathcal{F}_{\tau_{i}}$-measurable bounded functions, $\left|X_{\tau}\right| Q$-integrable, and define

$$
Y_{0} \equiv 0 \quad \text { and } \quad Y_{n} \equiv \sum_{i=0}^{n-1} U_{i}\left(X_{\tau_{i+1}}-X_{\tau_{i}}\right) \quad \text { for } n \geq 1
$$

Then

(a) $\left\{Y_{n}\right\}_{n \geq 0}$ is a martingale w.r.t. $\mathcal{F}_{\tau}=\left\{\mathcal{F}_{\tau_{i}}\right\}_{i \geq 0}$ and $Q$.

Assume further that $M_{\tau}$ is a $\mathcal{F}_{\tau}$-stopping time and that $M_{\tau}$ is bounded or the sequence $\left|Y_{n}\right|$ is bounded uniformly by an integrable function. Then, for any $k \geq 0$,

(b) $\mathbb{E}_{Q}\left[\sum_{i=k}^{M_{\tau}-1} U_{i}\left(X_{\tau_{i+1}}-X_{\tau_{i}}\right) \mid \mathcal{F}_{\tau_{k}}\right]=0$.

Proof. For (a), fix $n \geq 0$, then it holds that

$$
\mathbb{E}_{Q}\left[Y_{n+1} \mid \mathcal{F}_{\tau_{n}}\right]=\mathbb{E}_{Q}\left[\sum_{i=0}^{n} U_{i}\left(X_{\tau_{i+1}}-X_{\tau_{i}}\right) \mid \mathcal{F}_{\tau_{n}}\right]=\sum_{i=0}^{n-1} U_{i}\left(X_{\tau_{i+1}}-X_{\tau_{i}}\right)=Y_{n},
$$

since for $0 \leq i \leq n, U_{i}, X_{\tau_{i}}$ are $\mathcal{F}_{\tau_{i}}$-measurable, so $\mathbb{E}_{Q}\left[\left(X_{\tau_{n+1}}-X_{\tau_{n}}\right) \mid \mathcal{F}_{\tau_{n}}\right]=0$.

For (b), first observe that $Z_{n} \equiv \sum_{i=k}^{n-1} U_{i}\left(X_{\tau_{i+1}}-X_{\tau_{i}}\right)=Y_{n}-Y_{k}$ for $n>k$, and $Z_{n} \equiv 0$ if $0 \leq n \leq k$ is also a martingale w.r.t. $\mathcal{F}_{\tau}$.

For $m \geq 1$, consider $\sigma_{m}: \Omega_{0}(\tau) \rightarrow \mathbb{N}$ defined as follows:

$$
\sigma_{m}(\omega)= \begin{cases}k & \text { if } M_{\tau}(\omega)<k+1 \\ M_{\tau}(\omega) & \text { if } k+1 \leq M_{\tau}(\omega) \leq m \\ m & \text { if } k+1 \leq m<M_{\tau}(\omega)\end{cases}
$$

$\sigma_{m}$ is clearly bounded, and also a $F_{\tau}$-stopping time; this follows from

$$
\begin{aligned}
\left\{\sigma_{m} \leq n\right\}=( & \left.\{k \leq n\} \cap\left\{M_{\tau}<k+1\right\}\right) \\
& \cup\left(\left\{M_{\tau} \leq n\right\} \cap\left\{M_{\tau} \geq k+1\right\} \cap\left\{M_{\tau} \leq m\right\}\right) \\
& \cup\left(\{m \leq n\} \cap\left\{M_{\tau}>m\right\}\right) .
\end{aligned}
$$

If $n \leq k$, the second and third sets in the union are empty, and the first one belongs to $\mathcal{F}_{\tau_{n}}$. For $k+1 \leq n<m$, the third set is empty, the first is in $\mathcal{F}_{\tau_{n}}$, and

$$
\left.\left.\left\{M_{\tau} \leq n\right\} \cap\left\{M_{\tau} \geq k+1\right\} \cap\left\{M_{\tau} \leq m\right\}\right)=\left\{M_{\tau} \leq n\right\} \cap\left\{M_{\tau} \leq k+1\right\}\right) \in \mathcal{F}_{\tau_{n}} .
$$

On the other hand, if $k+1 \leq m \leq n$ the first and third sets belong clearly to $\mathcal{F}_{\tau_{n}}$, and

$$
\left.\left.\left\{M_{\tau} \leq n\right\} \cap\left\{M_{\tau} \geq k+1\right\} \cap\left\{M_{\tau} \leq m\right\}\right)=\left\{M_{\tau} \geq k+1\right\} \cap\left\{M_{\tau} \leq m\right\}\right) \in \mathcal{F}_{\tau_{m}} \subset \mathcal{F}_{\tau_{n}} .
$$

It follows, from [17, Prop. 1.83], using the stopping time $\sigma_{0} \equiv k \leq \sigma_{m}$, that

$$
\mathbb{E}_{Q}\left[Z_{\sigma_{m}} \mid \mathcal{F}_{\tau_{k}}\right]=\mathbb{E}_{Q}\left[\sum_{i=k}^{\sigma_{m}-1} U_{i}\left(X_{\tau_{i+1}}-X_{\tau_{i}}\right) \mid \mathcal{F}_{\tau_{k}}\right]=\mathbb{E}_{Q}\left[Y_{\sigma_{m}}-Y_{k} \mid \mathcal{F}_{\tau_{k}}\right]=0 .
$$


Observe that if $M_{\tau}$ is bounded then $Z_{M_{\tau}}=Z_{\sigma_{m}}$ for some $m \geq 1$. In general, $Z_{\sigma_{m}} \rightarrow Z_{M_{\tau}}$ pointwise, and from our hypotheses it follows that $\left|Z_{\sigma_{m}}\right|$ is bounded by an integrable function, so we have

$$
\mathbb{E}_{Q}\left[\sum_{i=k}^{M_{\tau}-1} U_{i}\left(X_{\tau_{i+1}}-X_{\tau_{i}}\right) \mid \mathcal{F}_{\tau_{k}}\right]=\lim _{m \rightarrow \infty} \mathbb{E}_{Q}\left[Z_{\sigma_{m}} \mid \mathcal{F}_{\tau_{k}}\right]=0 .
$$

Lemma 5. For a given $\omega \in \Omega_{0}(\tau)$ and $k \geq 0$, the set $\Omega_{\omega, k}(\tau)$, defined in Theorem 3 , belongs to $\mathcal{F}_{\tau_{k}}$.

Proof. We have to show that $A=\Omega_{\omega, k}(\tau) \cap\left\{\tau_{k} \leq t\right\} \in \mathcal{F}_{\tau_{k}}$, for all $t \geq 0$. Setting $C_{\omega, k}=C \cap\left[0, \tau_{k}(\omega)\right]$, observe that $\Omega_{\omega, k}(\tau)$ can be decomposed in the following way:

$$
\Omega_{\omega, k}(\tau)=\left(\bigcap_{s \in C_{\omega, k}}\left\{\omega^{\prime}: X_{s}\left(\omega^{\prime}\right)=X_{s}(\omega)\right\}\right) \cap\left(\bigcap_{i=0}^{k}\left\{\omega^{\prime}: \tau_{i}\left(\omega^{\prime}\right)=\tau_{i}(\omega)\right\}\right) .
$$

Note that if $t<\tau_{k}(\omega)$, then $\left\{\omega^{\prime}: \tau_{k}\left(\omega^{\prime}\right)=\tau_{k}(\omega)\right\} \cap\left\{\tau_{k} \leq t\right\}=\emptyset$, thus $A=\emptyset \in \mathcal{F}_{\tau_{k}}$. Consequently it is enough to consider $\tau_{k}(\omega) \leq t$. Let $s \in C_{\omega, k}$; it follows that $s \leq t$ and

$$
\left\{\omega^{\prime}: X_{s}\left(\omega^{\prime}\right)=X_{s}(\omega)\right\}=X_{s}^{-1}\left(\left\{X_{s}(\omega)\right\}\right) \in \mathcal{F}_{s} \subset \mathcal{F}_{t} .
$$

Therefore $X_{s}^{-1}\left(\left\{X_{s}(\omega)\right\}\right) \in \mathcal{F}_{\tau_{k}}$. On the other hand, since for $0 \leq i \leq k, \tau_{i} \leq \tau_{k}$ then $\tau_{i}$ are $\mathcal{F}_{\tau_{k}}$-measurable [21], which concludes that $\Omega_{\omega, k}(\tau) \in \overline{\mathcal{F}}_{\tau_{k}}$.

Acknowledgments. S. Ferrando would like to thank Zsolt Bihary and H. Föllmer for stimulating discussions.

\section{REFERENCES}

[1] B. Acciaio, M. Beiglböck, F. Penker and W. Schachermayer. A model-free version of the fundamental theorem of asset pricing and the super-replication theorem, Math. Finance 26 (2016), no. 2, 233-251. MR 3481303

[2] A. Alvarez, S. Ferrando and P. Olivares. Arbitrage and hedging in a non probabilistic framework. Math. Finan. Econ. 7 (2013), no. 1, 1-28. MR 3023889

[3] A. Alvarez and S. Ferrando. Trajectory-based models, arbitrage and continuity. Int. J. Theor. Appl. Finance 19 (2016), no. 3, 1650015, 34 pp. MR 3489932

[4] C. Bender, T. Sottinen and E. Valkeila. Fractional processes as models in stochastic finance. In: Advanced Mathematical Methods for Finance, 75-103. Springer-Verlag, 2011. MR 2792076

[5] M. Britten-Jones and A. Neuberger. Arbitrage pricing with incomplete markets, Appl. Math. Finance 3 (1996), no. 4, 347-363.

[6] M. Burzoni, M. Frittelli and M. Maggis. Universal arbitrage aggregator in discrete-time markets under uncertainty, Finance Stoch. 20 (2016) no. 1, 1-50. MR 3441285

[7] M. Burzoni, M. Frittelli and M. Maggis. Model-free superhedging duality, Ann. Appl. Probab. 27 (2017), no. 3, 1452-1477. MR 3678476

[8] P. Cheridito, M. Kupper and L. Tangpi. Duality formulas for robust pricing and hedging in discrete time, SIAM J. Financial Math. 8 (2017), no. 1, 738-765. MR 3705784

[9] A. M. G. Cox, Z. Hou, J. Obłój. Robust pricing and hedging under trading restrictions and the emergence of local martingale models, Finance Stoch. 20 (2016), no. 3, 669-704. MR 3519165 
[10] N. J. Cutland and A. Roux. Derivative Pricing in Discrete Time, Springer Undergraduate Mathematics Series. Springer, London, 2012. MR 3014681

[11] R. C. Dalang, A. Morton and W. Willinger. Equivalent martingale measures and no-arbitrage in stochastic securities market models, Stochastics Stochastic Rep. 29 (1990), no. 2, 185-201. MR 1041035

[12] I. Degano. Arbitraje y Cubrimiento. Enfoque no Probabilístico, Tesis doctoral, Facultad de Ciencias Exactas y Naturales, Universidad Nacional de Mar del Plata, 2018.

[13] I. Degano, S. E. Ferrando and A. González. Trajectory based market models. Evaluation of minmax price bounds, Dyn. Contin. Discrete Impuls. Syst. Ser. B Appl. Algorithms 25 (2018), no. 2, 97-128. MR 3775971

[14] S. Desmettre, R. Korn, P. Ruckdeschel and Frank T. Seifried. Robust worst-case optimal investment, OR Spectrum 37 (2015), no. 3, 677-701. MR 3359060

[15] R. A. Jarrow, P. Protter and H. Sayit. No arbitrage without semimartingales, Ann. Appl. Probab. 19 (2009), no. 2, 596-616. MR 2521881

[16] I. Karatzas and C. Kardaras. The numéraire portfolio in semimartingale financial models, Finance Stoch. 11 (2007), no. 4, 447-493. MR 2335830

[17] P. Medvegyev. Stochastic integration theory, Oxford Graduate Texts in Mathematics, 14. Oxford University Press, Oxford, 2007. MR 2345169

[18] R. Merton. Theory of rational option pricing. Bell J. Econom. Management Sci. 4 (1973), 141-183. MR 0496534

[19] M. Nutz. Superreplication under model uncertainty in discrete time, Finance Stoch. 18 (2014), no. 4, 791-803. MR 3255751.

[20] F. Riedel. Financial economics without probabilistic prior assumptions, Decis. Econ. Finance 38 (2015), no. 1, 75-91. MR 3325302

[21] A. N. Shiryaev. Optimal Stopping Rules. Stochastic Modelling and Applied Probability, 8. Springer-Verlag, 2008. MR 2374974.

[22] M. S. Taqqu and W. Willinger. The analysis of finite security markets using martingales, Adv. Appl. Probab. 19 (1987), no. 1, 1-25. MR 0876534.

\section{S. E. Ferrando}

Department of Mathematics, Ryerson University, 350 Victoria St., Toronto, Ontario M5B 2K3,

Canada

ferrando@ryerson.ca

\section{A. L. González $z^{凶}$}

Departamento de Matemática. Facultad de Ciencias Exactas y Naturales. Universidad Nacional de Mar del Plata. Funes 3350, B7600 Mar del Plata, Argentina

algonzal@mdp.edu.ar

I. L. Degano

Departamento de Matemática. Facultad de Ciencias Exactas y Naturales, Universidad Nacional de Mar del Plata. CONICET. Funes 3350, B7600 Mar del Plata, Argentina

ivandegano@mdp.edu. ar

M. Rahsepar

Department of Mathematics, Ryerson University, 350 Victoria St., Toronto, Ontario M5B 2K3, Canada

a.rasehpar@hotmail.com

Received: January 14, 2018

Accepted: September 21, 2018 